\title{
¿Cómo ha evolucionado la presión fiscal de la empresa española en la última década? Remedios normativos frente a la planificación fiscal
}

\author{
Juan Monterrey Mayoral ${ }^{a}$, Amparo Sánchez Segura ${ }^{\mathrm{b}}$ \\ a, b) Universidad de Extremadura; Departamento de Economía Financiera y Contabilidad, Avenida de Elvas, Badajoz, Spain.
}

${ }^{a}$ Corresponding author.

E-mail address: jmontrey@unex.es

\section{A R T I C L E I N F O}

\section{Article history:}

Received 2 April 2019

Accepted 8 July 2019

Available online 1 July 2020

\section{Códigos JEL:}

M40

H25

H26

Palabras clave:

Planificación fiscal

Tipos impositivos efectivos

\section{R E S U M E N}

En este trabajo hemos analizado la evolución de la presión fiscal soportada por la empresa española en la última década (2008-2017). Con base en una muestra de 31.966 observaciones empresas-años y empleando medidas empíricas de tributación efectiva inmunes al efecto de la variación del tipo impositivo estatutario operada a partir de 2015, dejamos constancia de una significativa aproximación gradual de los tipos reales a los nominales, convergencia que es especialmente apreciable en el segmento de las grandes empresas, a pesar de desplegar estrategias de planificación fiscal más eficientes que las de menor tamaño. Nuestros hallazgos revelan que tales estrategias se han visto claramente neutralizadas y dominadas con éxito por las medidas tributarias adoptadas en estos años, tendentes a diferir la deducibilidad fiscal de determinados gastos y pérdidas y a acelerar la recaudación. En definitiva, la evidencia aquí documentada, que se mantiene ante diferentes extensiones y pruebas empíricas complementarias, no se compadece con la extendida creencia acerca de la baja tributación de las empresas españolas, señaladamente las de gran dimensión.

(C)2020 ASEPUC. Published by EDITUM - Universidad de Murcia. This is an open access article under the CC BY-NC-ND license (http://creativecommons.org/licenses/by-nc-nd/4.0/).

How has the tax burden on Spanish companies evolved over the last decade? Regulatory remedies versus tax planning

A B S T R A C T

In this paper the evolution of the tax burden supported by Spanish companies in the last decade (2008-2017) has been analysed. Based on a sample of 31,966 company-year observations and using empirical measures of effective taxation that are immune to the effect of the variation in the statutory tax rate operated from 2015 onwards, we record a significant gradual approximation of the real rates to the nominal rates, a convergence that is particularly noticeable in the large company segment, despite deploying more efficient tax planning strategies than those of a smaller size. Our findings reveal that such strategies have been clearly neutralized and successfully dominated by the tax measures adopted in these years, aimed at deferring the tax deductibility of certain expenses and losses and accelerating tax collection. To sum up, the evidence documented in this paper, which is supported by various extensions and complementary empirical evidence, does not match the widespread belief about the low taxation of Spanish companies, particularly those of a large size.

(C)2020 ASEPUC. Publicado por EDITUM - Universidad de Murcia. Este es un artículo Open Access bajo la licencia CC BY-NC-ND (http://creativecommons.org/licenses/by-nc-nd/4.0/). 


\section{Introduction}

El presente trabajo tiene por objeto llevar a cabo un análisis empírico de la evolución de la presión fiscal soportada por las empresas españolas en la última década (2008-2017), con especial referencia a las de mayor tamaño. Con ello pretendemos contribuir a clarificar la polémica existente sobre su tributación real, periódicamente avivada con las publicaciones de los informes anuales de recaudación de la Agencia Tributaria y que se polariza entre quienes, de un lado, comparten con Conde-Ruiz (2015) que "la percepción popular de que las grandes empresas en general, independientemente del sector al que pertenezcan, pagan pocos impuestos, puede tener algo de justificación", participando así de una corriente en la que se integran buena parte de la literatura académica sobre planificación fiscal -anglosajona en su práctica totalidad-- o instituciones como la OCDE, y quienes, como organizaciones profesionales españolas de expertos fiscales (AEDAF, 2017) o empresariales (CEOE, 2017; Feito, 2018), sostienen que la metodología de cálculo del tipo impositivo efectivo que publica la Agencia Tributaria en los precitados informes de recaudación contiene errores, lo que daría lugar a mostrar una imagen distorsionada de la tributación de las empresas, sesgando a la baja la presión fiscal realmente soportada. En síntesis, las críticas a la Agencia Tributaria son metodológicas y se centran en el modo de tratar en sus informes la deducción por doble imposición de dividendos y la compensación de bases imponibles negativas.

Tampoco contribuye a clarificar la cuestión el incesante cambio normativo, especialmente acusado desde el comienzo de la crisis económica en 2008 y culminado con la reforma del Impuesto sobre Sociedades, tras la entrada en vigor en 2015 de la Ley 27/2014. El Servicio de Estudios del Registro de Economistas Asesores Fiscales ha identificado hasta 200 impactos fiscales en el impuesto en la última década ${ }^{1}$, habiendo sido modificado, al menos, por 12 textos legales de importancia, con cambios tan significativos como el tratamiento fiscal de los deterioros de cartera, de los resultados derivados de su enajenación, las limitaciones a la deducibilidad de los gastos financieros y al aprovechamiento de las bases imponibles negativas o el diferimiento de la deducibilidad de las amortizaciones. Todas estas medidas, si bien algunas de ellas temporales, han tenido una especial incidencia en las grandes empresas, que son las que se han venido situando en el centro de las críticas sobre su supuesta baja tributación.

El estudio de la presión fiscal y sus determinantes ha sido profusamente abordado en la literatura académica, estando bien documentado cómo se ve afectada por las características de las empresas, tanto las de carácter financiero (Shackelford \& Shevlin, 2001; Hanlon \& Heitzman, 2010), como las de otra naturaleza, estrategia y modelo de negocio (Higgins et al., 2015), su estructura de propiedad (Chen et al., 2010; Badertscher et al., 2013), la calidad de sus prácticas de gobierno (Desai et al., 2007; Richardson et al., 2013), el tipo de auditor (Kanagaretnam et al., 2016) o la modalidad de retribución de sus directivos (Armstrong et al., 2012).

El caso de las empresas españolas ha sido abordado por varios autores, que han aplicado diversas metodologías, abarcado distintos periodos temporales y contemplado diferentes ámbitos. Así, Álvarez García et al. (2004) analizan la evolución de la presión fiscal en la década de los años 90 -entre 1991 y 1998--; Calvé Pérez et al. (2005), Molina Llopis (2005) y Moreno Rojas et al. (2017) los determinantes

${ }^{1}$ https: / /economistas.es/cust notas/madrid-10-de-julio-de-2018-losexpertos-contabilizan-200-cambios-en-el-impuesto-sobre-sociedades-en-laultima-decada-2 de la presión fiscal soportada en función de las diferentes características corporativas de las empresas; los dos primeros estudios, en las compañías de reducida dimensión de la Comunidad Valenciana entre 1992 y 1999 , aportando evidencia de cómo la reforma fiscal de 1996 dio lugar a una reducción de la carga tributaria soportada por las empresas de reducida dimensión, y los terceros en las empresas del sector turístico para el periodo 2008-2013. En todos los casos la evidencia empírica deja clara constancia de una notable diversidad en los tipos impositivos efectivos de las empresas.

Asimismo, Fernández Rodríguez \& Martínez Arias (2008a, 2008b), para el periodo 1998-2003, estudian la presión fiscal en función tanto de la filiación sectorial de las empresas como de la comunidad autónoma en la que están asentadas; Fonseca Díaz et al. (2011), las diferencias existentes en la imposición efectiva soportada por bancos y cajas de ahorro entre 1993 y 2004, ligeramente superior en los primeros; Monterrey \& Sánchez Segura (2010), y Bona-Sánchez et al. (2019) documentan la mayor presión fiscal soportada por las empresas familiares frente a las de otras estructuras de propiedad; los primeros para los ejercicios 2002-2006 y los segundos para el periodo 2003-2013. Muy recientemente, Fernández Rodríguez et al. (2019), para los ejercicios entre 2008 y 2014, han aportado evidencia de cómo las empresas privadas muestran tipos efectivos superiores a las empresas públicas.

Por último, Fernández Rodríguez (2002, 2004a) y Monterrey \& Sánchez Segura (2015) abordan el estudio de los determinantes de la imposición efectiva en las empresas cotizadas; los primeros, para los años 1993-1998, ponen de manifiesto la sensibilidad de la presión fiscal ante el endeudamiento, la rentabilidad y la estructura del activo, mientras que los segundos, para los ejercicios 2008-2012, muestran cómo los esquemas retributivos de la alta dirección y el grado de independencia del Consejo de Administración influyen en la carga fiscal soportada.

Nuestro trabajo aborda, como hemos avanzado, el análisis empírico de la imposición efectiva de las empresas españolas en los últimos diez años, ya que consideramos que esta perspectiva temporal nos permitirá obtener una visión de conjunto de la evolución en el tiempo de un fenómeno muy interesante, en qué medida las estrategias de planificación fiscal adoptadas por las empresas españolas, por otra parte legítimas si están ubicadas dentro del marco normativo, dominan o no sobre las numerosas iniciativas de la Administración emprendidas en los últimos años con la finalidad de reforzar el cumplimiento de las obligaciones fiscales y acelerar la recaudación tributaria.

En una línea similar al nuestro, únicamente nos constan los muy recientes estudios empíricos llevados a cabo por Drake et al. (2018), Dyreng et al. (2017) y Edwards et al. (2019), y que por razón de su proximidad comentaremos sucintamente. En ellos se examina la evolución del tipo impositivo efectivo de las empresas cotizadas norteamericanas, documentando un significativo descenso en los últimos años, explicado, en cierta medida, por las propias características de las empresas y por la reducción generalizada en los tipos impositivos estatutarios de las jurisdicciones foráneas en las que operan las firmas multinacionales de Estados Unidos.

El de Dyreng et al. (2017) es el trabajo que cabe considerar como iniciador de esta línea de estudios longitudinales, y en que se aborda el estudio de los cambios operados en el tipo impositivo efectivo (siguiendo criterios de caja) entre 1998 y 2012, dejando constancia del descenso de la presión fiscal soportada en el curso de los 25 años contemplados y documentando cómo, en contra de la creencia generalizada, esta reducción de la carga tributaria es similar en las compañías 
multinacionales y las domésticas, que atribuyen a los cambios producidos en las características de las empresas, señaladamente la creciente intensidad de los activos intangibles y de los resultados no recurrentes, y al paulatino descenso de los tipos impositivos observado en otros países ${ }^{2}$.

Drake et al. (2018) profundizan en el estudio de Dyreng et al. (2017), amplían el periodo de análisis al comprendido entre 1996 y 2015 y concluyen que el declive en los tipos efectivos, con arreglo a criterios de carga fiscal devengada traería causa en una combinación de factores, entre los que se encontrarían el elevado nivel de beneficios obtenido por las compañías multinacionales norteamericanas procedentes de sus filiales extranjeras -que al soportar tipos más reducidos que el doméstico dan lugar a una reducción en el tipo efectivo medio de gravamen sobre el resultado consolidado-, la disminución operada en el tipo estatutario de algunos estados y atributos corporativos como la filiación sectorial el crecimiento o el tamaño empresarial.

Finalmente, el estudio de Edwards et al. (2019) también tiene por finalidad avanzar en la evidencia documentada por Dyreng et al. (2017), consideran el periodo 1996-2015 y, tomando el tipo efectivo según criterio de caja como objeto de estudio, obtienen evidencia de que los impuestos satisfechos no guardan una proporción estricta con los resultados antes de impuestos, como cabría esperar. Según los autores, esta ausencia de proporcionalidad no cabría atribuirla a las actividades de planificación fiscal seguidas por las empresas, sino a la tendencia creciente del resultado antes de impuestos.

Nuestro trabajo se suma a la reciente investigación empírica que acabamos de comentar sobre la tributación efectiva de la empresa española y contribuye, desde una perspectiva temporal, a un mejor entendimiento de las cargas fiscales soportadas por las firmas, a clarificar el debate sobre su grado de cumplimiento y a aportar evidencia sobre el modo en que las autoridades fiscales han conseguido combatir, a base de remedios normativos, las prácticas tributarias de las compañías.

El resto del trabajo se organiza como sigue, en la sección segunda planteamos el diseño de nuestra investigación, enunciando las hipótesis, especificando los modelos econométricos que nos permitirán abordar el contraste empírico de aquéllas y la selección de las variables que los integran; en la tercera sección presentamos la muestra de empresas, los principales estadísticos descriptivos y las correlaciones entre las variables objeto de análisis; en la cuarta mostramos y discutimos los principales resultados empíricos obtenidos; en la quinta llevamos a cabo pruebas complementarias que nos permitan extender nuestros resultados principales y en la sexta recogemos las principales conclusiones, así como las limitaciones que podrían afectar a la evidencia aquí documentada.

\section{Diseño de la investigación}

\subsection{Planteamiento de hipótesis}

Para Dyreng et al. (2017), es sorprendente la completa ausencia de estudios que analicen empíricamente la evolución temporal de los tipos impositivos efectivos a nivel microeconómico, puesto que, en su opinión, este enfoque aporta una enriquecedora perspectiva del modo en que aquéllos van modificándose en función de las características de las empresas y de los cambios en el marco normativo. No obstante, tanto Dyreng et al. (2017) como Drake et al. (2018) parecen obviar

${ }^{2}$ A diferencia de Estados Unidos, donde el tipo estatutario federal se ha mantenido inalterado entre 1998 y 2012, periodo estudiado por Dyreng et al. (2017). el hecho de que la presión fiscal soportada por las empresas es la resultante final de dos fuerzas que actúan en sentido opuesto, de una parte, las prácticas de planificación fiscal de las empresas ${ }^{3}$, más o menos agresivas y asumiendo mayores o menores niveles de riesgo, con la finalidad de soportar, como objetivo de primer orden, la menor carga fiscal actual, mediante la generación de ingresos no computables fiscalmente, inversiones en activos de tributación bonificada, y adoptando decisiones reales operativas que den lugar a ventajas fiscales, y como objetivo de segundo orden, diferir la deuda tributaria a ejercicios futuros, ya sea imputando fiscalmente ingresos contables a periodos posteriores, ya sea imputando al ejercicio corriente gastos fiscalmente deducibles que serán gastos contables de ejercicios futuros.

Frente a estas estrategias, también es legítimo que en el marco constitucional y garantizando la seguridad jurídica de los contribuyentes, la Administración tributaria emplee su potestad para adoptar medidas orientadas a garantizar el correcto cumplimiento de las obligaciones fiscales, a mejorar la normativa y a mitigar la crisis recaudatoria inducida por las muy adversas circunstancias económicas que han venido afectando a nuestro país, aproximando en la medida de lo posible los tipos impositivos reales a los nominales .

Así las cosas, y teniendo en cuenta que tanto la planificación fiscal de las empresas como las políticas tributarias de la Administración han de considerarse actividades continuadas en el tiempo, reviste interés verificar empíricamente cual de ambos efectos, la planificación fiscal o los remedios normativos de la Administración, es el dominante sobre el otro, y de aquí que la primera de las hipótesis que será objeto de contraste se enuncie como sigue,

H1, El tipo impositivo efectivo de las empresas españolas se ha reducido a lo largo de la última década

Ahora bien, el hecho de que nuestro análisis empírico confirme esta hipótesis podría ser simplemente una consecuencia mecánica de la evolución a la baja del tipo estatutario español, que en 2015 se redujo al $28 \%$ y a partir de 2016 al $25 \%$, con identificando erróneamente esta reducción con una mayor efectividad de las estrategias fiscales de las empresas. Por esta razón, entendemos que un enfoque alternativo, basado en examinar la aproximación entre los tipos nominal y efectivo del impuesto, puede aportar una mejor comprensión de la cuestión objeto de estudio, por lo que la segunda de nuestras hipótesis a contrastar será,

\section{H2, Las políticas tributarias de la Administración han dominado sobre la planificación fiscal de las em- presas españolas, por lo que se ha producido una con- vergencia entre los tipos nominal y efectivo del im- puesto a lo largo del tiempo}

En la medida en que los resultados empíricos confirmen esta hipótesis, el tipo efectivo se acercará paulatinamente del

\footnotetext{
${ }^{3}$ La planificación fiscal está muy bien definida en el apartado 12 del artículo 2 de la Resolución de 9 de febrero de 2016, del Instituto de Contabilidad y Auditoría de Cuentas, por la que se desarrollan las normas de registro, valoración y elaboración de las cuentas anuales para la contabilización del Impuesto sobre Beneficios, Son acciones que la entidad puede emprender, dentro del marco legal, para crear o incrementar ganancias fiscales en un determinado ejercicio, a través de la aplicación de las diferentes opciones o alternativas ofrecidas por la normativa fiscal, antes de que prescriba la posibilidad de deducir una pérdida fiscal $u$ otro crédito por operaciones anteriores, o para diferir beneficios a efectos fiscales. En el plano académico, la definición más extendida es la de Scholes et al. (2015), para quienes la planificación fiscal es el conjunto de prácticas que maximizan los flujos de efectivo esperados después de impuestos.
} 
estatutario, lo que indicará que las políticas de la Administración han dominado con éxito sobre las estrategias de planificación fiscal de las empresas. En caso contrario, es decir, de mayor divergencia entre el tipo nominal y el real, se tratará de una manifestación de menor eficacia de la Administración y/o de planificación fiscal eficiente.

\subsection{Modelos empíricos y especificación de variables para el contraste de las hipótesis}

Con la finalidad de contrastar empíricamente la primera de las hipótesis enunciadas, la evolución temporal de la presión fiscal soportada por las empresas españolas, emplearemos un modelo de regresión cuya expresión genérica es la siguiente,

$$
\text { PresiónFiscal }_{i t}=\alpha+\beta^{*} \mathrm{ANO}_{t}+\varepsilon_{i t}
$$

En la que, para cada empresa $i$ de la muestra y ejercicio $t$, PresiónFiscal ${ }_{i t}$ es la variable dependiente que representa la presión fiscal soportada, y $A \tilde{N} O_{t}$ es la variable explicativa que, como en el estudio de Dyreng et al. (2017), capta la tendencia temporal de la presión fiscal a lo largo de los ejercicios que comprende nuestra muestra de empresas, entre 2008 y 2017, y que toma el valor 1 para el ejercicio 2008, el valor 2 para 2009, y así sucesivamente hasta 2017, cuyo valor es 10 . De este modo, $A \tilde{N} O_{t}$ es una variable que va creciendo a medida que los ejercicios son más próximos al actual, y de aquí que un coeficiente $\beta$ negativo y estadísticamente significativo asociado a $A \tilde{N} O_{t}$ confirmaría la hipótesis e indicaría el declive paulatino del tipo impositivo efectivo entre 2008 y 2017.

Por su posible influencia sobre los resultados empíricos, creemos necesario detenernos en la descripción de las variables que hemos seleccionado como representativas de la presión fiscal, que es, como ya hemos comentado, el principal reproche que se formula a la Agencia Tributaria sobre su cómputo de la carga fiscal efectivamente soportada por las empresas. La literatura sobre planificación fiscal reconoce unánimemente la dificultad de medición de la presión fiscal, empleándose para ello diferentes medidas que vienen a captar, a su vez, distintas dimensiones de ésta.

Según nuestro parecer, dichas medidas pueden dividirse en dos categorías genéricas; la primera de ellas es la que agrupa las que tienen por objeto captar la presión tributaria soportada mediante la estimación del tipo impositivo efectivo, que combinan en el numerador una variable representativa del coste fiscal y en el denominador un subrogado o aproximación de la base imponible, ya que ésta no es observable. La segunda categoría aglutina las medidas que tienen por finalidad estimar la mayor o menor agresividad de las estrategias de planificación fiscal de las empresas a través del denominado book tax gap, mediante la diferencia entre resultado contable y una estimación de la base imponible, o bien mediante la diferencia entre tipo impositivo real y estatutario.

Una y otras, sin embargo, son medidas imperfectas por el hecho de estar basadas en estimaciones de la base imponible, y por esta razón, con la finalidad de dotar de mayor robustez a los hallazgos empíricos, el protocolo habitual en la investigación consiste en seleccionar más de una medida, puesto que los resultados documentados en la literatura son sensibles a la especificación de las variables representativas de los tipos reales. Así, por ejemplo, Lennox et al. (2012) emplean cinco medidas diferentes de tipos efectivos y dos de book tax gap; Chen et al. (2010), dos de las primeras y otras dos de las segundas, y Frank et al. (2009), una medida de tipo efectivo y dos de book tax gap.
Nosotros vamos a emplear cuatro medidas alternativas; las dos primeras, el tipo impositivo según el criterio de devengo y el tipo impositivo satisfecho según el criterio de caja, por ser las más empleadas en la literatura empírica y obtenidas, en ausencia de microdatos fiscales, a partir de la información contable de las empresas de nuestra muestra ${ }^{4}$, y la tercera y cuarta, dos versiones del book tax gap basada en la diferencia entre tipos reales y estatutarios, estimada una según bases de devengo y otra según caja.

La primera variable objeto de definición, el tipo impositivo efectivo devengado, generalmente se calcula de conformidad con lo dispuesto en el párrafo 86 de la NIC 12, es decir, como el cociente entre el gasto devengado por impuesto sobre beneficios y el resultado contable antes de impuestos, si bien dicha norma no especifica si el gasto devengado debe considerar o no, además del impuesto corriente, el diferido. Con relación al numerador, el impuesto devengado, entendemos que su especificación correcta pasa por incluir tanto el impuesto corriente como el diferido; su componente corriente, de acuerdo con lo establecido en la normativa contable, está minorado en el importe de las deducciones y ventajas fiscales que dan lugar a una reducción de la presión fiscal ${ }^{5}$, y la inclusión del importe diferido nos permite captar el impacto en la presión fiscal de las estrategias de planificación basadas en el diferimiento. Según nuestro parecer, si como definen Scholes et al. (2015), el objetivo de la planificación fiscal consiste en maximizar los flujos de efectivo presentes y futuros, ello se conseguirá explotando todas las posibilidades que ofrece la normativa tributaria, tanto reducir la base imponible del ejercicio corriente como para desplazar la presión fiscal a periodos futuros. Y de aquí que el gasto por impuesto devengado deba contemplar tanto su componente corriente como el diferido.

Asimismo, y dado que gasto por impuesto corriente se ve minorado como consecuencia de las deducciones por doble imposición por dividendos, y habida cuenta que dicho dividendo también ha sido sometido a tributación, aunque ello haya tenido lugar en sede de la empresa participada, desde una perspectiva de fondo económico lo conceptualmente correcto pasa por considerar la deducción por doble imposición como un mayor importe de impuesto devengado. Y con relación al denominador, la inclusión en el mismo del resultado contable antes de impuestos obedece a la necesidad de contemplar la presión fiscal como la parte de la renta generada que se destina a satisfacer la carga tributaria y no se pone a disposición de los propietarios, minorando la renta de éstos. Como sostiene Fernández Rodríguez (2004b), se trata del denominador correcto, puesto que si se desea conocer la repercusión que tiene este impuesto para la empresa en términos de carga fiscal, se debe tomar el resultado contable antes de que esté afectado por el propio tributo.

Por tanto, el tipo impositivo efectivo devengado es el siguiente,

$$
T I E_{-} D_{i t}=\left(I S_{i t}+\tau D_{i t}\right) / R C A I_{i t}
$$

Siendo TIE_ $D_{i t}$ el tipo impositivo efectivo, $I S_{i t}$ el impuesto total devengado, obtenido de la cuenta de pérdidas y ganan-

\footnotetext{
${ }^{4}$ Para una discusión detallada de los problemas asociados a la estimación de los tipos impositivos reales a partir de los datos contenidos en los estados financieros, remitimos al lector a los excelentes trabajos de Hanlon (2003) y Graham et al. (2012).

${ }^{5}$ El apartado 3 del artículo 2 de la Resolución de 9 de febrero de 2016, del Instituto de Contabilidad y Auditoría de Cuentas, por la que se desarrollan las normas de registro, valoración y elaboración de las cuentas anuales para la contabilización del Impuesto sobre Beneficios, dispone, Las deducciones y otras ventajas fiscales en la cuota del impuesto, excluidas las retenciones y pagos a cuenta, así como las pérdidas fiscales compensables de ejercicios anteriores y aplicadas efectivamente en éste, darán lugar a un menor importe del impuesto corriente.
} 
cias, $\tau$ el tipo del impuesto y $D_{i t}$ el importe de los ingresos procedentes de los dividendos, en aquellas empresas de la muestra en las que en el activo de su balance de situación figuren registradas inversiones en empresas del grupo, multigrupo y asociadas ${ }^{6}$, y $R C A I_{i t}$ el resultado contable antes de impuestos. Entendemos que de este modo logramos la mejor aproximación posible a la presión fiscal devengada, que, como acertadamente sostiene el informe de la AEDAF (2017), debe considerar la deducción por doble imposición como un ajuste positivo, aproximándose de este modo a la cuota íntegra minorada en el importe de deducciones y bonificaciones fiscales ${ }^{7}$.

Esta especificación difiere de la empleada por la Agencia Tributaria en sus informes, que calcula el tipo efectivo de dos maneras diferentes; la primera, como cociente entre la cuota líquida y el resultado contable antes de impuestos, y la segunda, como cociente entre la cuota líquida y la base imponible. En ambos casos, la inserción en el numerador de la cuota líquida, es decir, una vez deducidas del mismo las deducciones por doble imposición, da lugar a que ambos ratios combinen numerador y denominador heterogéneos y, en consecuencia, no comparables, ya que al tomar la cuota líquida, el denominador correcto serían en ambos casos el resultado antes de impuestos, minorado en el importe de los ingresos por dividendos a los que puede ser aplicada la deducción por doble imposición. Según nuestro parecer, el procedimiento de cálculo del tipo impositivo efectivo empleado por la Agencia Tributaria sesga a la baja la presión fiscal realmente soportada por las empresas.

Así pues, el modelo genérico [1] adoptará la expresión [2] siguiente,

$$
T I E \_D_{i t}=\alpha+\beta^{*} \mathrm{ANO}_{t}+\varepsilon_{i t}
$$

La segunda variable que emplearemos como medida de la presión fiscal es el tipo efectivo del impuesto pagado, TIE_ $C_{i t}$, que definimos como el cociente entre el Impuesto sobre Sociedades pagado, $I P_{i t}$, ajustado por el importe de los dividendos cobrados, obtenidos ambos del estado de flujos de efectivo, y el resultado contable antes de impuestos,

$$
T I E_{-} C_{i t}=\left(I P_{i t}+\tau D_{i t}\right) / R C A I_{i t}
$$

Y en consecuencia, el modelo [1] también se especificará del siguiente modo,

$$
T I E_{-} C_{i t}=\alpha+\beta^{*} \mathrm{ANO}_{t}+\varepsilon_{i t}
$$

La segunda de las hipótesis enunciadas será objeto de contraste mediante el modelo genérico siguiente,

$$
G A P_{i t}=\alpha+\beta^{*} \mathrm{ANO}_{t}+\varepsilon_{i t}
$$

En el que la variable dependiente, $G A P_{i t}$, es una medida del book tax gap que representa la distancia existente entre el tipo impositivo nominal y el real, y $A \tilde{N} O_{t}$, como en el modelo [1], caracteriza la tendencia temporal lineal. Así, un coeficiente $\beta$ negativo y estadísticamente significativo dejaría

${ }^{6}$ Dado que también pueden registrarse en la cuenta de pérdidas y ganancias dividendos procedentes de otras inversiones financieras en instrumentos de patrimonio que a las que no sea de aplicación la deducción por doble imposición de dividendos.

${ }^{7}$ No obstante, hay que tener en cuenta que la eliminación del impuesto subyacente (underlying tax) de los dividendos ha pasado por diferentes etapas a lo largo de la década; así, entre 2008 y 2014, se aplicó la deducción por doble imposición contemplada en el Real Decreto Legislativo 4/2004; en 2015 el mecanismo de exención previsto en la Ley 27/2014, y en 2016 y 2017, por aplicación del Real Decreto Ley 3/2016, la limitación de dicho mecanismo de exención al $50 \%$ de la cuota íntegra. constancia de que la divergencia entre el tipo estatutario y el real se ha visto reducida con el paso del tiempo, lo que confirmaría la hipótesis de que las políticas tributarias de la Administración dominan sobre la planificación fiscal de las empresas.

A su vez, expresaremos $G A P_{i t}$ como el porcentaje de la diferencia entre tipo estatutario y real con relación al tipo nominal, que para el tipo efectivo devengado será,

$$
G A P_{-} D_{i t}=\left(T I N_{i t}-T I E_{-} D_{i t}\right) / T I N_{i t}
$$

Siendo $\operatorname{TIN}_{t}$ el tipo impositivo nominal. De este modo, $G A P D_{i t}$ nos permite obtener una medida de la efectividad real de las prácticas de planificación fiscal, al normalizar el efecto de los cambios en el tipo estatutario. Así, por ejemplo, si el tipo impositivo efectivo de una empresa en el ejercicio 1 , TIE_ $D_{1}$, es el $26 \%$ y el nominal, $T I N_{1}$, es el $30 \%, G A P \_D_{1}$ alcanzará un valor de 0,13 [(30\%-26\%)/30\%], lo que significa que la empresa en cuestión soporta una presión fiscal un $13 \%$ inferior a la que le correspondería según su tipo nominal. Si en el periodo posterior 2 tiene lugar una reducción del tipo nominal, de manera que $T_{I N}$ es el $25 \%$ y, asimismo, su tipo efectivo, TIE_ $D_{2}$, es el $20 \%, G A P \_D_{2}$ será igual a 0,20 [(25\%-20\%)/25]. Como podemos apreciar, el hecho de que TIE_ $D_{1}$ sea el $26 \%$ y que TIE_ $D_{2}$ sea el $20 \%$ traslada la idea -correcta-- de que la presión efectivamente soportada se ha reducido de un ejercicio a otro, pero, paradójicamente, la divergencia entre tipos reales y nominales, y por tanto la agresividad fiscal de la compañía, ha aumentado del $13 \%$ al $20 \%$. Por tanto, el análisis de los tipos efectivos es un indicador de la presión fiscal, pero en un escenario de tipos nominales cambiantes se convierte en una medida sesgada de la efectividad de la planificación fiscal de las empresas, ya que, como acabamos de comentar, un aumento (o disminución) del gap puede ser consecuencia de una modificación en los tipos formales y no traer causa en cambios en las estrategias fiscales de las empresas, y de aquí que, a nuestro entender, $G A P D_{i t}$ sea el indicador correcto.

El̄o da lugar a la especificación [5] siguiente,

$$
G A P_{-} D_{i t}=\alpha+\beta^{*} \mathrm{ANO}_{t}+\varepsilon_{i t}
$$

$\mathrm{Y}$ para el caso del tipo efectivo pagado,

$$
G A P_{-} C_{i t}=\left(T I N_{i t}-T I E_{-} C_{i t}\right) / T I N_{i t}
$$

Y de aquí que el modelo [4] adopte también la siguiente forma econométrica,

$$
G A P_{-} C_{i t}=\alpha+\beta^{*} \mathrm{ANO}_{t}+\varepsilon_{i t}
$$

En nuestro estudio empírico emplearemos la metodología de datos de panel, para controlar la heterogeneidad inobservable de las empresas de la muestra, llevando a cabo nuestras estimaciones por efectos fijos, al ser la modalidad apropiada por rechazarse la hipótesis nula del test de Hausman de igualdad de coeficientes entre los modelos de efectos fijos y aleatorios.

\section{Muestra, estadísticos descriptivos y correlaciones en- tre variables}

En condiciones ideales, un estudio de estas características se habría acometido tomando los microdatos procedentes de las declaraciones tributarias de las empresas, pero la confidencialidad de las mismas lo impide llevar a cabo. Por esta razón, al análisis empírico no puede sino efectuarse sobre la base de la información procedente de las cuentas anuales 
de las empresas, y de aquí que la medición de la imposición efectiva no pueda ser nunca exacta y sea inevitable asumir este hecho (Hanlon, 2003; Blouin, 2014). Nuestra muestra de empresas ha sido configurada a partir de la base de datos $\mathrm{Sabi}^{\circ}$ y abarca, para los ejercicios comprendidos entre 2008 y 2017, las sociedades mercantiles domiciliadas en España, activas, auditadas, que formulan cuentas anuales normales individuales y pertenecen a todos los sectores económicos excepto financiero y bancario (CNAE 64), seguros (CNAE 65) y los CNAE 84 y 99, por desarrollar actividades cuyas peculiaridades aconsejan excluirlas de este trabajo.

El hecho de configurar la muestra con cuentas individuales y no consolidadas tiene por finalidad asegurar que todas las compañías que la integran están sometidas al mismo tipo nominal del impuesto y a idéntico marco normativo y sistema de enforcement, ya que una muestra formada a nivel de grupos consolidados integraría entidades con filiales que, al estar domiciliadas en otros países, no son sujetos pasivos del impuesto en España, siendo por tanto contribuyentes en otras jurisdicciones con diferentes regulaciones y tipos impositivos, lo que podría afectar considerablemente a nuestros resultados empíricos.

Además, hemos requerido que todas las observaciones presentes en la muestra tengan resultados antes de impuestos positivo, ya que, como indica Fernández Rodríguez (2004b), cuando éste es negativo los tipos efectivos calculados del modo en que hemos descrito podrían no ser reflejo de la presión fiscal realmente soportada por las empresas, si tanto el resultado antes de impuestos como el gasto (o el pago) por impuesto son negativos, el tipo efectivo resultante resulta ser positivo, pero en realidad la empresa no ha soportado ninguna presión fiscal, y si el resultado antes de impuestos es negativo y el gasto (o el pago) por impuesto es positivo, la empresa soporta presión fiscal pero el tipo efectivo que se obtiene es negativo.

También hemos excluido de la muestra todas las empresas en cuyo patrimonio neto figuran resultados negativos de ejercicios anteriores, para evitar el problema de interpretación que apunta Hanlon (2003), quien observa que la estimación de la base imponible a partir del resultado contable antes de impuestos contiene un inevitable error de medición en empresas con créditos fiscales, ya que éstos afectan al gasto devengado por impuesto, pero no a la base imponible. Asimismo, la OCDE (2003, capítulo 3, p. 25) considera que la medición del tipo efectivo, en presencia de pérdidas fiscales compensables, se complica como consecuencia de los problemas tanto teóricos como prácticos que plantea, ya que su aprovechamiento reduce el numerador del ratio en el ejercicio en el que ello tiene lugar, pero no en todos los casos, ya que en las empresas que reconocen contablemente el crédito fiscal derivado de la compensación futura de bases imponibles negativas este efecto no se produce. Y además, en las firmas que proceden a registrar contablemente el crédito fiscal que trae causa en la generación de bases imponibles negativas, el tipo efectivo resultante es negativo, lo que contamina por completo los resultados empíricos.

La muestra da comienzo en el ejercicio 2008 por tratarse del primero en el que las compañías españolas formularon sus cuentas anuales con arreglo al actual Plan General de Contabilidad, que incorporó a sus contenidos el estado de flujos de efectivo, y cuya información es necesaria para la configuración de parte de las variables objeto de estudio, y asimismo modificó sustancialmente el registro y valoración del impuesto sobre beneficios.

Como la longitud de nuestra serie temporal --diez ejercicios-- podría dar lugar a un posible sesgo de supervi- vencia, hemos permitido que las empresas puedan entrar y salir de la muestra, formando así una muestra variable integrada por todas las empresas que, reuniendo las características antes indicadas, cuentan con suficientes datos en un determinado ejercicio que permitan disponer de las variables necesarias. Con estos requerimientos, la muestra, una vez eliminados datos ilegibles o inexistentes, quedó configurada por 31.966 observaciones empresas-años ${ }^{8}$,

No obstante, la literatura sobre planificación fiscal es prácticamente unánime al señalar que estas estrategias se identifican mejor cuando son excluidas de las muestras las observaciones en las que el tipo impositivo efectivo, tanto expresado en términos de devengo, TIE $D_{i t}$, como de caja, TIE $C_{i t}$, discurre en un rango entre 0 y 1 , ya que los valores fuera de estos límites son difíciles de interpretar (Armstrong et al., 2012; Badertscher et al., 2013; Davis et al., 2016; Gallemore y Labro 2015). Además, como demuestran empíricamente De Simone et al. (2019), las estimaciones son más consistentes y robustas refinando la muestra censurando las variables en el sentido que acabamos de indicar ${ }^{9}$. Tras excluir las observaciones fuera de dicho rango, la muestra final quedó configurada por 31.891 observaciones para la variable $T I E \_D_{i t}$ y 31.364 para la variable TIE_ $C_{i t}$, quedando excluidas $\overline{75}$ y 602 , respectivamente.

La tabla 1 muestra los principales estadísticos descriptivos de las variables que emplearemos en nuestro análisis empírico, que mostramos en porcentajes para facilitar su interpretación (con la excepción de $T A M_{i t}$, expresada en logaritmo). La variable TIE $D_{i t}$, primera de las representativas de la presión fiscal, exhibe una media de 27,1\% (mediana, 28,7\%), lo que deja constancia de que entre 2008 y 2017, las empresas soportaron una presión fiscal generalmente inferior al tipo de gravamen nominal ${ }^{10}$, mientras que la desviación estándar, 7,7\%, indica una moderada dispersión. La segunda variable asociada a la carga fiscal soportada, TIE_ $C_{i t}$, arroja un valor medio de 26,6\% (mediana, 26,3\%), muy similares a los de TIE $D_{i t}$, pero con una dispersión muy superior, a tenor de su desviación estándar, 14, \%, lo que pone de relieve una mayor heterogeneidad, propia de las variables definidas en términos de caja con relación a las especificadas en términos de devengo.

En cuanto a las variables representativas de la distancia entre presión fiscal real y nominal, la primera de ellas, GAP $D_{i t}$, indica que, en promedio, las empresas de la muestra tienen un diferencial del 6,7\% (mediana $0,9 \%$ ) con relación al tipo estatutario, pero con una elevada dispersión, como indica una desviación estándar del 26,3\%. El examen de los valores de $G A P C_{i t}$, con media de $8,1 \%$ (mediana $7,7 \%$ ) y desviación estándar de 49,6\%, confirma la mayor heterogeneidad observada en las variables formuladas en términos de caja.

Con respecto a las restantes variables que emplearemos en nuestro posterior análisis empírico, el tamaño, $T A M_{i t}$, tiene un valor medio de 9,667 (mediana 9,448 ) y el endeudamiento, $E N D_{i t}$, de $51,7 \%$ (mediana $52,5 \%$ ). Con relación a las variables que captan el peso relativo de determinadas partidas del balance, $I N T_{i t}$ representa, en promedio, el 2,2\% del activo (mediana 0,1\%); $M A T_{i t}$, el 21,2\% (mediana 15,3\%) y $T E S_{i t}$, el 9,7\% (mediana 5,3\%). Por último la rentabilidad económica, $R O I_{i t}$, alcanza una media del 10,3\% (mediana

${ }^{8}$ Con la siguiente distribución, 2008, 3.096 observaciones; 2009, 3.059; $2010,3.345 ; 2011,3.409 ; 2012,3.233,2013$, 3.303; 2014, 3.295; 2015, $3.060 ; 2016,3.092$, у 2017, 3.074 .

${ }^{9}$ Aunque ello podría privarnos, como razonan Armstrong et al. (2015), de conocer el impacto de formas muy agresivas o muy conservadoras de planificación fiscal.

${ }^{10}$ Los tipos impositivos nominales fueron el $30 \%$ para los ejercicios comprendidos entre 2008 y 2014, el $28 \%$ para 2015 y el $25 \%$ para 2016 y 2017. 
Tabla 1

Estadísticos descriptivos

\begin{tabular}{cccccc}
\hline Variables & Media & $\begin{array}{c}\text { Desv. } \\
\text { estándar }\end{array}$ & $25 \%$ & $\begin{array}{c}50 \% \\
\text { (mediana) }\end{array}$ & $75 \%$ \\
\hline TIE_Dit & $27,1 \%$ & $7,7 \%$ & $24,6 \%$ & $28,7 \%$ & $30,0 \%$ \\
TIE_Cit & $26,6 \%$ & $14,4 \%$ & $18,2 \%$ & $26,3 \%$ & $31,6 \%$ \\
GAP_Dit & $6,7 \%$ & $26,3 \%$ & $-0,2 \%$ & $0,9 \%$ & $12,4 \%$ \\
GAP_C $C_{i t}$ & $8,1 \%$ & $49,6 \%$ & $-8,8 \%$ & $7,7 \%$ & $37,1 \%$ \\
TAM $_{i t}$ & 9,667 & 1,177 & 8,858 & 9,448 & 10,220 \\
END $_{i t}$ & $51,7 \%$ & $23,3 \%$ & $33,1 \%$ & $52,5 \%$ & $70,5 \%$ \\
INT & $2,2 \%$ & $8,3 \%$ & $0,0 \%$ & $0,1 \%$ & $0,6 \%$ \\
MAT $_{i t}$ & $21,2 \%$ & $20,6 \%$ & $4,6 \%$ & $15,3 \%$ & $31,5 \%$ \\
ROI $_{i t}$ & $10,3 \%$ & $14,6 \%$ & $3,4 \%$ & $7,1 \%$ & $13,0 \%$ \\
TES $_{i t}$ & $9,7 \%$ & $11,9 \%$ & $1,6 \%$ & $5,3 \%$ & $13,4 \%$ \\
\hline
\end{tabular}

La muestra está integrada por 31.966 observaciones empresas-años de sociedades mercantiles domiciliadas en España, activas, auditadas, que formulan cuentas anuales normales individuales y pertenecen a todos los sectores económicos excepto a los identificados como CNAE 64, 65, 84 y 99, con datos completos en, al menos, uno de los ejercicios comprendidos entre 2008 y 2017 y sin registrar resultados negativos de ejercios anteriores. Al requerir que las variables $T I E$ D y TIE $C_{\text {it }}$ muestren valores jercicios anteriores. Al requerir que 作 para el análisis empírico de los modelos que emplean las variables TIE_ $D_{i t}$ y GAP $D_{i t}$ y 31.364 observaciones para los que emplean las variables TIE_ $C_{i t}$ y $G A P{ }_{-} C_{i t}$. Para cada observación de la empresa $i$ y ejercicio $t, T I E \_D_{i t}$ es el tipo impositivo efectivo expresado como cociente entre el impuesto sobre beneficios devengado y el resultado contable antes de impuestos; $T I E C_{i t}$ es el tipo impositivo efectivo definido como cociente entre el impuesto sobre beneficios pagado y el resultado contable antes de impuestos; GAP $D_{i t}$ es la diferencia entre el tipo impositivo nominal y el tipo efectivo devengado, dividida por el tipo estatutario; GAP $D_{\text {it }}$ es la diferencia entre el tipo impositivo nominal y el tipo efectivo pagado, dividida por el tipo estatutario; $T A M_{i t}$ es el logaritmo las deudas totalel y el acivo las deudas totales y el activo total, ambos referidos al ciertion cociente entre el valor contable del inmovilizado intangible y el activo total, ambos al cierre del ejercicio; $I N M_{i t}$ es el cociente entre el valor contable del inmovilizado material y el activo total, ambos al cierre del ejercicio; $R O I_{i t}$ es el cociente entre el resultado contable antes de impuestos y el activo total, ambos al cierre del ejercicio, y $T E S_{i t}$ es el cociente entre la suma de tesorería y equivalente a tesorería y activo total, ambos al cierre de ejercicio. Salvo $T A M_{i t}$, que se expresa en logaritmo, las restantes variables se muestran en porcentajes.

\section{$7,1 \%)$.}

La tabla 2 ofrece las correlaciones entre las variables, siendo destacables las existentes entre TIE_D $D_{i t}$ y $T I E \_C_{i t}(8,6 \%$ Pearson y $36,4 \%$ Spearman) y GAP $D_{i t}$ y $G A P C_{i t}(\overline{9}, 5 \%$ Pearson y 33,5\% Spearman), que, a tenor de sus valores, vienen a corroborar la necesidad de contar tanto con variables basadas en el principio de devengo como en el criterio de caja. También son apreciables las correlaciones del tamaño, TAM ${ }_{i t}$, con $I N T_{i t}$, de signo positivo, y con $T E S_{i t}$, negativa, las negativas del endeudamiento, $E N D_{i t}$, con $R O I_{i t}$ y $T E S_{i t}$ y las de la tesorería, $T E S_{i t}$ con $R O I_{i t}$, positiva, y con $M A T_{i t}$, negativa. En general, las correlaciones son significativamente más elevadas al transformarse los valores de las variables en rangos para aplicar el test de Spearman. Por último, los reducidos valores de las correlaciones entre las variables independientes nos permiten descartar cualquier efecto de la multicolinealidad sobre los resultados empíricos posteriores.

\section{Resultados empíricos}

La tabla 3 muestra los resultados de la regresión de los modelos [2] y [3], en los que las variables dependientes son el tipo impositivo efectivo devengado, TIE_ $D_{i t}$, y el tipo efectivo según criterio de caja, TIE_ $C_{i t}$, respectivamente. Con relación al tipo devengado, TIE $D_{i t}^{-}$, el término constante es positivo, alcanzando un valor de 0,290 y siendo estadísticamente muy significativo, mientras que la variable independiente $A \tilde{N} O_{t}$ es negativa y también muy significativa, lo que deja constancia de que el tipo devengado ha tenido una evolución decreciente en el tiempo, reduciéndose por tanto la carga fiscal realmente soportada por las empresas a lo largo de la última
Tabla 2

Correlaciones entre variables

\begin{tabular}{|c|c|c|c|c|c|c|c|c|c|c|}
\hline & $T I E_{-} D_{i t}$ & $T I E_{-} C_{i t}$ & $G A P_{-} D_{i t}$ & $G A P_{-} C_{i t}$ & $T_{A A M_{i t}}$ & $E N D_{i t}$ & $I N T_{i t}$ & $M A T_{i t}$ & ROI It $_{\text {it }}$ & $T E S_{\text {it }}$ \\
\hline \multirow[t]{2}{*}{$T I E_{-} D_{i t}$} & 1,000 & $36,4 \%$ & $-88,5 \%$ & $-31,1 \%$ & $-8,6 \%$ & $13,3 \%$ & $-7,5 \%$ & $-5,7 \%$ & $-5,7 \%$ & $-2,9 \%$ \\
\hline & & 0,000 & 0,000 & 0,000 & 0,000 & 0,000 & 0,000 & 0,000 & 0,000 & 0,000 \\
\hline \multirow[t]{2}{*}{$T I E_{-} C_{i t}$} & $8,6 \%$ & 1,000 & $-32,8 \%$ & $-98,2 \%$ & $-5,0 \%$ & $0,9 \%$ & $-4,3 \%$ & $-3,6 \%$ & $-11,2 \%$ & $-0,3 \%$ \\
\hline & 0,000 & & 0,000 & 0,000 & 0,000 & 0,119 & 0,000 & 0,000 & 0,000 & 0,541 \\
\hline \multirow[t]{2}{*}{$G A P_{-} D_{i t}$} & $-99,6 \%$ & $-8,8 \%$ & 1,000 & $33,5 \%$ & $5,9 \%$ & $-11,7 \%$ & 7,3\% & $7,2 \%$ & $3,6 \%$ & $-0,4 \%$ \\
\hline & 0,000 & 0,000 & & 0,000 & 0,000 & 0,000 & 0,000 & 0,000 & 0,000 & 0,457 \\
\hline \multirow[t]{2}{*}{$G A P_{-} C_{i t}$} & $-9,1 \%$ & $-99,9 \%$ & $9,5 \%$ & 1,000 & $3,6 \%$ & $0,3 \%$ & $4,1 \%$ & $3,8 \%$ & $10,3 \%$ & $-1,1 \%$ \\
\hline & 0,000 & 0,000 & 0,000 & & 0,000 & 0,613 & 0,000 & 0,000 & 0,000 & 0,059 \\
\hline \multirow[t]{2}{*}{ TAM $_{i t}$} & $-2,9 \%$ & $-1,1 \%$ & $2,4 \%$ & $1,1 \%$ & 1,000 & $2,6 \%$ & $19,0 \%$ & $4,1 \%$ & $1,6 \%$ & $-18,8 \%$ \\
\hline & 0,000 & 0,045 & 0,000 & 0,049 & & 0,000 & 0,000 & 0,000 & 0,004 & 0,000 \\
\hline \multirow[t]{2}{*}{$E N D_{\text {it }}$} & $3,0 \%$ & $0,0 \%$ & $-2,5 \%$ & $0,1 \%$ & $1,0 \%$ & 1,000 & $1,2 \%$ & $-7,5 \%$ & $-36,3 \%$ & $-22,2 \%$ \\
\hline & 0,000 & 0,983 & 0,000 & 0,935 & 0,087 & & 0,032 & 0,000 & 0,000 & 0,000 \\
\hline \multirow[t]{2}{*}{$I N T_{i t}$} & $-0,1 \%$ & $-0,2 \%$ & $0,0 \%$ & $0,2 \%$ & $17,4 \%$ & $2,4 \%$ & 1,000 & $9,9 \%$ & $2,1 \%$ & $-6,3 \%$ \\
\hline & 0,833 & 0,719 & 0,968 & 0,729 & 0,000 & 0,000 & & 0,000 & 0,000 & 0,000 \\
\hline \multirow[t]{2}{*}{$M A T_{i t}$} & $-1,6 \%$ & $-0,4 \%$ & $1,8 \%$ & $0,5 \%$ & $8,0 \%$ & $-2,2 \%$ & $-7,5 \%$ & 1,000 & $-7,1 \%$ & $-15,0 \%$ \\
\hline & 0,004 & 0,445 & 0,001 & 0,422 & 0,000 & 0,000 & 0,000 & & 0,000 & 0,000 \\
\hline \multirow[t]{2}{*}{ ROI $_{\text {it }}$} & $-3,6 \%$ & $-1,5 \%$ & $3,5 \%$ & $1,5 \%$ & $-4,0 \%$ & $-19,4 \%$ & $2,0 \%$ & $-7,5 \%$ & 1,000 & $20,9 \%$ \\
\hline & 0,000 & 0,008 & 0,000 & 0,007 & 0,000 & 0,000 & 0,000 & 0,000 & & 0,000 \\
\hline \multirow[t]{2}{*}{ TES it } & $-0,8 \%$ & $-0,4 \%$ & $0,1 \%$ & $0,4 \%$ & $-13,7 \%$ & $-21,5 \%$ & $-5,9 \%$ & $-18,1 \%$ & $16,9 \%$ & 1,000 \\
\hline & 0,137 & 0,444 & 0,800 & 0,494 & 0,000 & 0,000 & 0,000 & 0,000 & 0,000 & \\
\hline
\end{tabular}

Características de la muestra y definición de variables en la tabla 1. Las correlaciones por rangos de Spearman se muestran en la parte superior de la diagonal y las de Pearson en la parte inferior, y están expresadas en porcentajes. Los valores $p$ de los coeficientes de correlación se ofrecen en cursiva.

década. Tomados en su conjunto estos resultados, cabe interpretar que en el año base o ejercicio de comienzo de nuestro análisis, 2008, la presión fiscal media soportada por las compañías de la muestra fue del $29 \%$, tipo efectivo que fue declinando muy ligeramente a lo largo del decenio analizado, a un ritmo medio anual del $0,4 \%$.

Tabla 3

Resultados de la regresión del modelo

$T I E_{-} D_{i t}\left(T I E_{-} C_{i t}\right)=\alpha+\beta^{*} \mathrm{AN} \mathrm{NO}_{t}+\varepsilon_{i t}$

\begin{tabular}{lcccc}
\hline & \multicolumn{2}{c}{ TIE_Dit } & \multicolumn{2}{c}{ TIE_Cit } \\
& Coeficientes & $t$ & Coeficientes & $t$ \\
\hline Constante & 0,290 & $39,97^{* * *}$ & 0,279 & $16,38^{* * *}$ \\
$A \tilde{N} O_{t}$ & $-0,004$ & $-24,58^{* * *}$ & $-0,003$ & $-8,46^{* * *}$ \\
& & & & \\
$\mathrm{R}^{2}$ & $8,6 \%$ & & $2,30 \%$ & \\
$\mathrm{~F}$ & $604,02^{* * *}$ & & $71,58^{* * *}$ & \\
$\mathrm{~N}$ & 31.891 & & 31.364 & \\
\hline
\end{tabular}

Características de la muestra y definición de las variables $T I E_{-} D_{i t}$ y $T I E_{-} C_{i t}$ en la tabla 1. La variable $\mathrm{AÑO}_{t}$ es la diferencia entre el ejercicio fiscal $t$ al que pertenece cada observación menos el número 2007, pudiendo tomar valores entre 1 (ejercicio 2008) y 10 (ejercicio 2017). La significación estadística se expresa en asteriscos, a niveles superiores al $90 \%\left(^{*}\right)$, $\left.95 \%{ }^{(* *}\right)$ y $99 \%\left({ }^{* * *}\right)$ de probabilidad. Errores estándar niveles superiores al $90 \%\left({ }^{*}\right), 95 \%\left({ }^{* *}\right)$ y $99 \%\left({ }^{* *}\right)$ de probabilidad. Errores estándar corregidos por
et al., 2010).

Con respecto al modelo que incorpora como variable dependiente el tipo efectivo pagado, TIE_ $C_{i t}$, los resultados obtenidos son muy similares, ya que el término constante es positivo y muy significativo $(0,279)$ y la variable explicativa $A \tilde{N} O_{t}$, a tenor de su signo negativo $(-0,003)$ y de su elevada significación, pone asimismo de manifiesto una paulatina reducción de la presión fiscal. En suma, esta evidencia viene a corroborar que entre 2008 y 2017 y en promedio, el tipo efectivo pagado se ha visto reducido a un ritmo anual del $0,3 \%$, partiendo de un $27,9 \%$ en 2008 . Los coeficientes de determinación de ambos modelos, $8,6 \%$ y $2,30 \%$, son ligeramente superiores a los documentados por Drake et al. (2018), Dyreng et al. (2017) y Edwards et al. (2018) en Estados Unidos. 
Así pues, esta evidencia confirma la primera de las hipótesis enunciadas y pone de manifiesto la disminución gradual del gravamen fiscal entre 2008 y 2017 . No obstante, los resultados de la regresión de los modelos [2] y [3] que acabamos de comentar podrían estar críticamente afectados por las modificaciones operadas en el tipo impositivo estatutario tras la entrada en vigor de la Ley 27/2014. En efecto, entre los ejercicios 2008 y 2014 el tipo general del Impuesto sobre Sociedades se mantuvo en el $30 \%$, para experimentar una reducción al $28 \%$ en 2015 y situarse en el $25 \%$ en 2016 y 2017. Con la finalidad de verificar en qué medida el declinar de la presión tributaria real entre 2008 y 2018 que hemos descrito podría obedecer a dichos cambios, en lugar de ser la consecuencia de una mayor agresividad fiscal de las compañías, en la tabla 4 recogemos los resultados obtenidos en la regresión de los modelos [5] y [6], en los que, como antes hemos indicado, las variables dependientes, GAP_D $D_{i t}$ y $G A P \_C_{i t}$, captan las diferencias existentes, en términos porcentuales, entre el tipo impositivo estatutario y el efectivamente devengado y pagado, respectivamente. La principal ventaja de operar con estas variables es que al estar expresadas como porcentajes de las diferencias entre tipo estatutario y efectivo con relación a éste último, sus valores numéricos están normalizados y no se ven afectados por las alteraciones del tipo nominal, permitiendo la comparabilidad en el tiempo de la mayor o menor proximidad entre ambos.

\section{Tabla 4}

Resultados de la regresión del modelo $G A P_{-} D_{i t}\left(G A P_{-} C_{i t}\right)=\alpha+\beta^{*} \mathrm{ANO}_{t}+\varepsilon_{i t}$

\begin{tabular}{lcccc}
\hline & \multicolumn{2}{c}{$G A P_{-} D_{i t}$} & \multicolumn{2}{c}{$G A P_{-} C_{i t}$} \\
& Coeficientes & $t$ & Coeficientes & $t$ \\
\hline Constante & 0,087 & $28,23^{* * *}$ & 0,122 & $20,68^{* * *}$ \\
$A \tilde{N} O_{t}$ & $-0,004$ & $-7,43^{* * *}$ & $-0,009$ & $-7,88^{* * *}$ \\
$\mathrm{R}^{2}$ & & & & \\
$\mathrm{~F}$ & $7,1 \%$ & & $2,60 \%$ & \\
$\mathrm{~N}$ & $95,288^{* * *}$ & & $62,11^{* * *}$ & \\
\hline
\end{tabular}

Características de la muestra y definición 2 de las variables $G A P D_{i t}$ y $G A P C_{i t}$ en la tabla 1 y de $\mathrm{ANNO}_{t}$ en la tabla 3. La significación estadística se expresa en asteriscos, a niveles superiores al $90 \%\left({ }^{*}\right), 95 \%\left({ }^{* *}\right)$ y $99 \%\left({ }^{* * *}\right)$ de probabilidad. Errores estándar corregidos por dependencia en sección cruzada y serie temporal (Petersen, 2009; Gow et al., 2010).

Como podemos apreciar, los resultados obtenidos de la regresión del modelo [5] empleando GAP_ $D_{i t}$ como variable dependiente aportan un valor positivo de 0,087 para el término constante y negativo de $-0,004$ para la variable explicativa, $A \tilde{N} O_{t}$, siendo ambos estadísticamente muy significativos. Esta evidencia indica que en el ejercicio en el que da comienzo nuestro análisis, 2008, el tipo impositivo real era, en promedio, un 8,7\% inferior al nominal, reduciéndose paulatinamente a un ritmo del $0,4 \%$ anual hasta 2017. Muy similares son los resultados que arroja el modelo [6], en el que empleando GAP_ $C_{i t}$ como variable dependiente, se obtienen unos valores de $\overline{0}, 122$ para el término constante y de -0,009 para $A \tilde{N} O_{t}$ ambos muy significativos, lo que indica que con el paso del tiempo se ha reducido paulatinamente la diferencia entre tipo estatutario e impuesto pagado, partiendo en 2008 de un tipo pagado inferior en un 12,2\% al nominal y acortándose la diferencia entre ambos en un $0,9 \%$ promedio anual hasta 2017. Los coeficientes de determinación son muy similares a los obtenidos en la tabla 3 anterior.

Así pues, esta segunda evidencia que mostramos en la tabla 4 implica que, al sustituir las variables TIE_ $D_{i t}$ y TIE_ $C_{i t}$ por GAP $D_{i t}$ y GAP $C_{i t}$, respectivamente, que eliminan el posible efecto en los resultados empíricos de los cambios en los tipos nominales, se pone de manifiesto que en la última década, la empresa española ha ido acercando su tributación por el Impuesto sobre Sociedades a sus tipos nominales, lo que viene a confirmar la segunda de las hipótesis que hemos planteado. A tenor de estos hallazgos, y según nuestro parecer, las críticas vertidas a las empresas no parecen tener sustento empírico, y, es más, nos permiten arribar exactamente a la conclusión contraria, es decir, que en la última década se ha producido un claro proceso de convergencia de los tipos nominales y reales del impuesto, y ello con independencia de las reducciones operadas en los primeros.

A la vista de los resultados obtenidos, parece conveniente detenernos en el hecho de que el coeficiente $\beta$ asociado a la variable explicativa $A \tilde{N} O_{t}$ alcance en el modelo [6] un valor de 0,009, más del doble que el 0,004 obtenido en el modelo [5]. Ello significa que mientras la convergencia entre tipos nominales y reales según criterios de devengo se ha reducido a un ritmo anual del 0,4\%, la aproximación de ambos tipos según criterios de caja ha sido mucho más acelerada, un $0,9 \%$ en cada ejercicio, lo que podría ser la consecuencia de que en los últimos años las autoridades fiscales han adoptado medidas tendentes a acelerar la recaudación por este tributo, entre otras la limitación a la deducibilidad de gastos financieros, de amortizaciones y los pagos fraccionados mínimos. En otras palabras, la presión fiscal determinada según criterios de caja sería superior a la calculada con arreglo a criterios de devengo como consecuencia de la anticipación en el pago del impuesto instituida por la Administración tributaria, y, de ser así, los balances de las empresas captarían este fenómeno registrando un montante de activos por impuestos diferidos creciente en el tiempo.

Para verificar este extremo, hemos obtenido los resultados de la regresión del modelo [7] siguiente,

$$
A I D_{i t}\left(P I D_{i t}\right)=\alpha+\beta^{*} \mathrm{ANO}_{t}+\varepsilon_{i t}
$$

En el que $A I D_{i t}$ es el importe de los activos por impuestos diferidos registrados en el balance de situación, deflactado por el valor contable del activo total al cierre de ejercicio, y que es la expresión contable de las diferencias temporarias entre resultado contable y base imponible que, tratándose de gastos, no han sido objeto de deducción en el ejercicio corriente o anteriores y que podrán ser aplicadas y deducidas en ejercicios posteriores, o que, siendo ingresos, su incorporación a la base imponible ha tenido lugar con anterioridad a su reconocimiento contable ${ }^{11}$. Asimismo, y para ilustrar al lector, también obtendremos los resultados de la regresión [7] anterior empleando como variable dependiente $P I D_{i t}$, que es el montante de pasivos por impuestos diferidos reconocidos en balance, dividido por el importe del activo a cierre de ejercicio. Los pasivos por impuestos diferidos surgen como consecuencia de integrar en la base imponible ingresos en ejercicios posteriores a aquel en el que tiene lugar su reconocimiento contable, o de imputar en la base imponible gastos en periodos anteriores al de su registro contable.

Como podemos ver, los resultados de la regresión del modelo [7] tomando $A I D_{i t}$ como variable dependiente indican, a tenor del signo positivo y la significación estadística tanto del

\footnotetext{
${ }^{11}$ En el caso de las empresas de nuestra muestra, los activos por impuestos diferidos también contienen deducciones pendientes de aplicar en ejercicios posteriores como consecuencia de insuficiencia de cuotas, pero es muy improbable que incluyan créditos fiscales generados por bases imponibles negativas pendientes de compensación, ya que, como indicábamos en la sección 3 de este trabajo, todas las observaciones corresponden a empresas con resultado contable positivo y sin resultados negativos de ejercicios anteriores.
} 
Tabla 5

Resultados de la regresión del modelo $A I D_{i t}\left(P I D_{i t}\right)=\alpha+\beta^{*} A \tilde{N} O_{t}+\varepsilon_{i t}$

$$
A I D_{i t}
$$

PID it

\begin{tabular}{lcccc} 
& Coeficientes & $t$ & Coeficientes & $t$ \\
\hline Constante & 0,003 & $17,96^{* * *}$ & 0,007 & $41,76^{* * *}$ \\
$A \tilde{N} O_{t}$ & 0,002 & $12,10^{* * *}$ & $-0,001$ & $-3,49^{* * *}$ \\
& & & & \\
$\mathrm{R}^{2}$ & $4,56 \%$ & & $3,40 \%$ & \\
$\mathrm{~F}$ & $146,46^{* * *}$ & & $12,18^{* * *}$ & \\
$\mathrm{~N}$ & 31.966 & & 31.966 & \\
\hline
\end{tabular}

Características de la muestra en la tabla 1 y de $\mathrm{AÑO}_{t}$ en la tabla 3. Para cada observación de la empresa $i$ y ejercicio $t, A I D_{t}$ es el cociente entre los activos por impuestos diferidos y el activo total, ambos al cierre del ejercicio, y $P I D_{t}$ es el cociente entre los pasivos por impuestos diferidos y el activo total, ambos al cierre del ejercicio. La significación estadística se expresa en asteriscos, a niveles superiores al $90 \%\left({ }^{*}\right), 95 \%$ $\left({ }^{* *}\right)$ y $99 \%\left({ }^{* * *}\right)$ de probabilidad. Errores estándar corregidos por dependencia en sección cruzada y serie temporal (Petersen, 2009; Gow et al., 2010).

término constante como de $A \tilde{N} O_{t}$, que los activos por impuestos diferidos han ido adquiriendo un mayor peso relativo en el balance de las empresas españolas con el paso del tiempo, y ello como consecuencia, fundamentalmente, de anticipar el pago del impuesto con relación al momento de su devengo. Así, mientras en el ejercicio inicial de nuestra muestra, 2008, los activos por impuestos diferidos representaban el 0,3\% del activo total, su peso relativo ha ido aumentando a una tasa media anual del 0,2\%, lo que, con relación al 0,3\% del años base, nos parece una tasa de crecimiento ciertamente notable.

Asimismo, los resultados del modelo [7] tomando $P I D_{i t}$ como variable dependiente ponen de relieve que las estrategias de diferimiento de impuestos de las compañías españolas van reduciéndose con el paso del tiempo, como indica con toda claridad el coeficiente asociado a $A \tilde{N} O_{t}$, que es negativo y estadísticamente muy significativo, -0,001. En este caso, los pasivos por impuestos diferidos suponían en 2008 el 0,7\% --más del doble que los activos de la misma naturaleza-y se han visto reducidos en un $0,1 \%$ en cada ejercicio.

Contemplada en su conjunto, la evidencia empírica que hemos documentado en esta sección no se compadece con las manifestaciones acerca de que las empresas españolas van reduciendo paulatinamente la presión fiscal que soportan como consecuencia de sus estrategias tributarias. Es cierto que los resultados obtenidos de las regresiones de las ecuaciones [2] y [3] trasladan la idea de una menor carga tributaria soportada, pero se trata, simplemente, de la consecuencia mecánica de la reducción del tipo estatutario desde el $30 \%$ al $25 \%$, puesto que la estimación de los parámetros de las ecuaciones [5] y [6] nos permite identificar una clara aproximación de los tipos reales a los nominales, que es el objetivo abiertamente manifestado por la Administración tributaria ${ }^{12}$ y que refutaría la extendida idea de que la empresa española lleva a cabo prácticas de elusión fiscal. Asimismo, la evolución temporal de activos y pasivos por impuestos diferidos confirmaría que la estrategia de aceleración de la recaudación adoptada por las autoridades fiscales da lugar a que el impuesto pagado vaya aumentando con el paso del tiempo con relación al devengado.

\footnotetext{
${ }^{12}$ Como se menciona en los informes anuales de recaudación de la Agencia Tributaria y, también, en los preámbulos de la Ley 27/2014, de 27 de noviembre, del Impuesto sobre Sociedades, y del Real Decreto-ley 3/2016, de 2 de diciembre, por el que se adoptan medidas en el ámbito tributario dirigidas a la consolidación de las finanzas públicas y otras medidas urgentes en materia social.
}

\section{Extensiones y pruebas complementarias}

Con la finalidad de complementar los resultados que hemos discutido en la sección anterior, seguidamente llevaremos a cabo algunas extensiones que nos permitirán reforzar y dar robustez a los resultados obtenidos, así como aportar algunas evidencias adicionales de interés. Con tal finalidad, analizaremos el posible efecto que las características corporativas de las empresas de nuestra muestra pueden ejercer sobre la presión fiscal soportada, la sensibilidad de ésta ante la entrada en vigor de la Ley 27/2014, que constituye el cambio normativo de mayor calado producido en los años recientes y la reestimación de nuestros resultados para el caso de las empresas españolas de gran dimensión.

\subsection{El efecto de las características corporativas sobre la carga fiscal soportada}

Está ampliamente descrita en la literatura empírica la influencia que determinadas características empresariales ejercen sobre la carga fiscal soportada. Con el objeto de verificar en qué medida la creciente aproximación entre los tipos nominales y los reales que hemos documentado pudieran ser una consecuencia de dichas características y de los cambios operados en ellas a lo largo de la década estudiada, seguidamente replicaremos las regresiones de nuestros modelos empíricos [5] y [6] incorporando a los mismos los atributos más empleados en los diferentes estudios sobre la materia, bien razonados en Graham et al. (2012), Hanlon \& Heitzman (2010) y Shackelford \& Shevlin (2001). Como sostienen Dyreng et al. (2017), estos rasgos idiosincrásicos podrían afectar a nuestros resultados, habida cuenta de que a lo largo de la década que estamos contemplando como horizonte temporal de nuestro análisis, las empresas podrían actuar hoy de un modo diferente a como lo hicieron diez años atrás.

Para ello consideraremos el tamaño, el nivel de endeudamiento, la intensidad de intangibles, el peso relativo del inmovilizado material, la rentabilidad económica y el riesgo fiscal, que son variables convencionalmente asociadas a las diferencias en la carga fiscal soportada. El primero de ellos, el tamaño, ha mostrado resultados contradictorios con la planificación fiscal, ya que, si bien las empresas de mayor dimensión pueden destinar mayores recursos a esta tarea (Dyreng et al., 2008), o, como sostiene Conde-Ruiz (2015), acceder a mayores beneficios fiscales, no es menos cierto que cuanto mayor es el tamaño de las compañías mayor es también su visibilidad, por lo que algunos autores, como Desai \& Dharmapala (2006), Dyreng et al. (2016), Gallemore et al. (2014) y Guenther et al. (2019), sostienen que en el segmento de las empresas muy grandes es posible que limiten sus estrategias fiscales para evitar incurrir en significativos costes de agencia o en riesgos reputacionales, limitándose a desarrollar prácticas de planificación subóptimas.

Con respecto al endeudamiento, la teoría de la estructura de capital, encabezada por Modigliani \& Miller (1963) y continuada, entre otros, por De Angelo \& Masulis (1980), implica que, ceteris paribus, los incentivos para financiar las inversiones con deuda aumentan con el tipo impositivo marginal, debido a la deducibilidad fiscal de los intereses. Esta predicción teórica surge del hecho de que la retribución a los acreedores financieros, los intereses de la deuda, son fiscalmente deducibles, a diferencia de la retribución a los accionistas, los dividendos, que no tienen ninguna ventaja o reducción fiscal. Bajo este planteamiento, las empresas tendrían, en principio, incentivos para financiar sus inversiones con deuda, dado que su mejor fiscalidad favorece la creación 
de valor para los propietarios de la firma, por lo que cabe esperar una relación negativa entre nivel de endeudamiento y carga tributaria soportada.

La intensidad de intangibles podría, en el caso español, explicar una mayor divergencia entre tipos impositivos reales y nominales, habida cuenta de los incentivos fiscales existentes que darían lugar a una reducción de los tipos reales. Asimismo, el peso relativo del inmovilizado material ha sido considerado, entre otros, en los trabajos de Fernández Rodríguez (2004a) y Gupta \& Newberry (1997) como factor mitigante de la presión fiscal. En cuanto a la rentabilidad, está descrito en numerosos estudios, entre otros los de Fernández Rodríguez (2004a) y Chen et al. (2010), cómo las compañías con mayor rentabilidad tendrán mayores incentivos para desplegar estrategias de reducción de sus cargas tributarias, mostrando una mayor divergencia entre tipos nominales y reales. Por último, el riesgo fiscal también podría explicar la conducta de las empresas en el orden tributario y tratarse de un elemento moderador de prácticas fiscales más agresivas (Monterrey \& Sánchez Segura, 2015).

De este modo, los modelos [5] y [6] adoptarán la siguiente expresión,

$$
\begin{aligned}
G A P_{-} D_{i t}=\alpha+ & \beta_{1}^{*} \mathrm{ANO}_{t}+\beta_{2}^{*} T A M_{i t}+\beta_{3}^{*} E N D_{i t}+\beta_{4}^{*} I N T_{i t} \\
& +\beta_{5}^{*} M A T_{i t}+\beta_{6}^{*} R O I_{i t}+\beta_{7}^{*} T E S_{i t}+\varepsilon_{i t}
\end{aligned}
$$

$$
\begin{aligned}
G A P_{-} C_{i t}=\alpha+ & \beta_{1}^{*} \mathrm{ANO}_{t}+\beta_{2}^{*} T A M_{i t}+\beta_{3}^{*} E N D_{i t}+\beta_{4}^{*} I N T_{i t} \\
& +\beta_{5}^{*} M A T_{i t}+\beta_{6}^{*} R O I_{i t}+\beta_{7}^{*} T E S_{i t}+\varepsilon_{i t}
\end{aligned}
$$

En los que $T A M_{i t}$ es el tamaño, expresado como el logaritmo neperiano del activo total al cierre del ejercicio; $E N D_{i t}$ es el endeudamiento, caracterizado como cociente entre las deudas totales y el activo total, ambos referidos al cierre del ejercicio; $I N T_{i t}$ es la intensidad de los intangibles, definida como cociente entre el valor contable del inmovilizado intangible y el activo total, ambos al cierre del ejercicio; $I N M_{i t}$ es la intensidad del inmovilizado material, expresada como cociente entre el valor contable del inmovilizado material y el activo total, ambos al cierre del ejercicio; $R O I_{i t}$ es la rentabilidad, aproximada por el cociente entre el resultado contable antes de impuestos y el activo total, ambos al cierre del ejercicio, y TES $S_{i t}$ es el riesgo fiscal, subrogado por la posición de liquidez de la empresa (Hanlon et al., 2013) y cuyo valor numérico es el cociente entre la suma de tesorería y equivalente a tesorería y activo total, ambos al cierre de ejercicio. Asimismo, los modelos [8] y [9] incorporan los habituales controles de la filiación sectorial de cada compañía, a un nivel de profundidad de dos dígitos del CNAE.

La tabla 6 siguiente recoge los resultados de la regresión de los modelos [8] y [9]. Al emplear GAP_D $D_{i t}$ como variable dependiente, $A \tilde{N} O_{t}$ mantiene el signo negativo y la significación estadística que venimos documentando, por lo que cabe interpretar que, tras controlar dichas características, la convergencia entre el tipo devengado real y el nominal es un hallazgo robusto. Asimismo, el tamaño de la empresa, $T A M_{i t}$, arroja un coeficiente positivo y significativo, indicando que las compañías españolas de mayor dimensión son más propensas a incurrir en prácticas de planificación fiscal y exhiben un mayor gap, mientras que el nivel de endeudamiento, $E N D_{i t}$, es negativo y significativo, tal como pronosticábamos, y ello a pesar de la limitación de la deducibilidad de los gastos financieros vigente desde 2012.

También han resultado ser significativos y con el signo esperado los coeficientes asociados a la intensidad de los inmovilizados materiales, $M A T_{i t}$, y a la rentabilidad, $R O I_{i t}$, ambos
Tabla 6

Resultados de la regresión del modelo GAP_ $D_{i t}\left(G A P_{-} C_{i t}\right)=\alpha+\beta_{1}^{*} \mathrm{AÑO}_{t}+$ $\beta_{2}^{*} T A M_{i t}+\beta_{3}^{*} E N D_{i t}+\beta_{4}^{*} I N T_{i t}+\beta_{5}^{*} M A T_{i t}+\beta_{6}^{*} R O I_{i t}+\beta_{7}^{*} T E S_{i t}+\varepsilon_{i t}$

\begin{tabular}{lcccc}
\hline & \multicolumn{2}{c}{ GAP_Dit } & \multicolumn{2}{c}{ GAP_C $C_{i t}$} \\
& Coeficientes & $t$ & Coeficientes & $t$ \\
\hline Constante & $-0,023$ & $-1,77^{*}$ & $-0,090$ & $-3,54^{* *}$ \\
AN $O_{t}$ & $-0,005$ & $-9,40^{* * *}$ & $-0,008$ & $-8,04^{* * *}$ \\
TAM $_{i t}$ & 0,015 & $11,68^{* * *}$ & 0,017 & $7,15^{* * *}$ \\
END $_{i t}$ & $-0,084$ & $-12,78^{* * *}$ & 0,017 & 1,40 \\
$I N T_{i t}$ & $-0,025$ & $-1,41$ & $-0,056$ & $-1,62$ \\
MAT $_{i t}$ & 0,044 & $6,03^{* * *}$ & 0,053 & $3,79^{* * *}$ \\
ROI $_{i t}$ & 0,093 & $9,02^{* * *}$ & 0,276 & $13,98^{* * *}$ \\
TES $_{i t}$ & $-0,026$ & $-2,00^{* *}$ & $-0,027$ & $-1,08$ \\
$\mathrm{R}^{2}$ & $16,80 \%$ & & $10,22 \%$ & \\
$\mathrm{~F}$ & $57,11^{* * *}$ & & $45,66^{* * *}$ & \\
$\mathrm{~N}$ & 31.891 & & 31.364 & \\
\hline
\end{tabular}

Características de la muestra y definición de variables en la tabla 1. La significación estadística se expresa en asteriscos, a niveles superiores al $90 \%\left({ }^{*}\right), 95 \%\left({ }^{* *}\right)$ y $99 \%$ $\left.{ }^{* * *}\right)$ de probabilidad. Errores estándar corregidos por dependencia en sección cruzada y serie temporal (Petersen, 2009; Gow et al., 2010). Para simplificar la lectura, no se muestran los coeficientes asociados a sectores económicos.

positivos, y con signo negativo la posición de liquidez, $T E S_{i t}$. Por el contrario, la intensidad de los intangibles, $I N T_{i t}$, en los que se encuentran registradas las actividades de I+D y los fondos de comercio, no ha resultado se estadísticamente significativa, lo que en nuestra opinión es atribuible a la escasa importancia de estos elementos con relación al activo total (en promedio representan únicamente el 2,2\% de éste).

Empleando GAP_ $C_{i t}$ como variable dependiente, el ejercicio fiscal, $A \tilde{N} O_{t}$, el tamaño, $T A M_{i t}$, la intensidad de inmovilizados materiales, $M A T_{i t}$, y la rentabilidad, $R O I_{i t}$, mantienen el signo y la significación estadística, pero se desvanecen las del endeudamiento, $E N D_{i t}$, y la liquidez, $T E S_{i t}$. Así pues, nuestros resultados dejan constancia de que, tras controlar la posible influencia de las características corporativas, se mantiene una significativa aproximación de los tipos impositivos reales a los nominales; en el caso del modelo [8], con GAP $D_{i t}$ como variable dependiente, el coeficiente $\beta_{1}$ asociado a $A \tilde{N} O_{t}$, $-0,005$, significa que, permaneciendo constantes las restantes variables contempladas en el modelo, en el curso de una década se obtendría un acercamiento del tipo real al estatutario del 5\%, y en el modelo [9], cuya variable dependiente es $G A P C_{i t}$, el valor 0,008 del coeficiente $\beta_{1}$ vinculado a $A \tilde{N} O_{t}$ implica una aproximación del tipo real al nominal aún mayor, el $8 \%$.

\subsection{Efectos de la reforma fiscal de 2015 sobre la presión tributaria}

Como antes hemos comentado, a lo largo de la década hemos asistido a una ininterrumpida sucesión de cambios normativos, por lo que no es posible aislar para su estudio el efecto de una concreta medida sobre la presión fiscal. Por esta razón nos vamos a centrar en el análisis del impacto de la reforma de mayor calado, la operada en 2015 con la precitada Ley 27/2014. Con tal finalidad, en los modelos [10] y [11] siguientes hemos sustituido la variable experimental $A \tilde{N} O_{t}$ por $R E F O R M A_{t}$, que toma el valor 1 para las observaciones de la muestra que sean posteriores al ejercicio $2015 \mathrm{y}$ 0 en caso contrario, 


$$
\begin{gathered}
G A P_{-} D_{i t}=\alpha+\beta_{1}^{*} R E F O R M A_{t}+\beta_{2}^{*} T A M_{i t}+\beta_{3}^{*} E N D_{i t} \\
+\beta_{4}^{*} I N T_{i t}+\beta_{5}^{*} M A T_{i t}+\beta_{6}^{*} R O I_{i t}+\beta_{7}^{*} T E S_{i t}+\varepsilon_{i t} \\
G A P_{-} C_{i t}=\alpha+\beta_{1}^{*} R E F O R M A_{t}+\beta_{2}^{*} T A M_{i t}+\beta_{3}^{*} E N D_{i t} \\
+\beta_{4}^{*} I N T_{i t}+\beta_{5}^{*} M A T_{i t}+\beta_{6}^{*} R O I_{i t}+\beta_{7}^{*} T E S_{i t}+\varepsilon_{i t}
\end{gathered}
$$

La tabla 7 recoge los resultados de las regresiones de ambos modelos, en los que podemos comprobar cómo la nueva variable de tratamiento, $R E F O R M A_{t}$, tras controlar las variables representativas de las características de las empresas de la muestra, ha resultado ser negativa y muy significativa, lo que indica que la reforma del Impuesto sobre Sociedades en 2015 tuvo éxito al conseguir aproximar los tipos efectivos a los nominales.

Tabla 7

Resultados de la regresión del modelo

$G A P_{-} D_{i t}\left(G A P_{-} C_{i t}\right)=\alpha+\beta_{1}^{*} R E F O R M A_{t}+\beta_{2}^{*} T A M_{i t}+\beta_{3}^{*} E N D_{i t}+$ $\beta_{4}^{*} I N T_{i t}+\beta_{5}^{*} \bar{M} A T_{i t}+\beta_{6}^{*} R O I_{i t}+\beta_{7}^{*} T E S_{i t}+\varepsilon_{i t}$

\begin{tabular}{lcccc}
\hline & \multicolumn{2}{c}{$G A P_{-} D_{i t}$} & \multicolumn{2}{c}{$G A P_{-} C_{i t}$} \\
& Coeficientes & $t$ & Coeficientes & $t$ \\
\hline Constante & $-0,075$ & $-5,43^{* * *}$ & $-0,148$ & $-5,62^{* * *}$ \\
REFORMA $^{* *}$ & $-0,031$ & $-9,23^{* * *}$ & $-0,028$ & $-4,20^{* * *}$ \\
TAM $_{i t}$ & 0,015 & $11,67^{* * *}$ & 0,017 & $6,79^{* * *}$ \\
END $_{i t}$ & $-0,082$ & $-12,55^{* * *}$ & 0,023 & $1,83^{*}$ \\
INT $_{i t}$ & $-0,028$ & $-1,59^{*}$ & $-0,061$ & $-1,76^{*}$ \\
MAT $_{i t}$ & 0,045 & $6,10^{* * *}$ & 0,054 & $3,89^{* * *}$ \\
ROI $_{i t}$ & 0,096 & $9,25^{* * *}$ & 0,279 & $14,18^{* * *}$ \\
TES $_{i t}$ & $-0,024$ & $-1,88^{*}$ & $-0,034$ & $-1,35$ \\
$\mathrm{R}^{2}$ & $16,70 \%$ & & $8,78 \%$ & \\
$\mathrm{~F}$ & 76,71 & & $38,95^{* * *}$ & \\
$\mathrm{~N}$ & 31.891 & & 31.364 & \\
\hline
\end{tabular}

Características de la muestra y definición de variables en la tabla 1. La variable $R E F O R M A_{t}$ toma el valor 1 si las observaciones pertenecen al ejercicio 2015 o posterior, y 0 en caso contrario. La significación estadística se expresa en asteriscos, a niveles superiores al $90 \%\left({ }^{*}\right), 95 \%\left({ }^{* *}\right)$ y $99 \%\left({ }^{* * *}\right)$ de probabilidad. Errores estándar corregidos por dependencia en sección cruzada y serie temporal (Petersen, 2009 ; Gow corregidos por sectores económicos.

\subsection{Planificación fiscal en las grandes empresas}

Como hemos comentado en secciones precedentes, es en el ámbito de las grandes empresas donde se han centrado las críticas a una supuesta reducida tributación, y asimismo, los resultados empíricos que hemos comentado dejan constancia de que el gap entre tipos reales y nominales es tanto mayor cuanto mayor es también el tamaño de las compañías. No obstante, algunos autores, como Desai \& Dharmapala (2006), Dyreng et al. (2016) y Guenther et al. (2019) sostienen que es posible que las empresas muy grandes limiten sus estrategias fiscales para evitar incurrir en significativos costes de agencia o en riesgos reputacionales. Por esta razón, consideramos de interés profundizar en el estudio de la carga fiscal en el subgrupo de las empresas de mayor dimensión de nuestra muestra, para lo que hemos reestimado los parámetros de la regresión del modelo [8], cuya variable dependiente es $G A P \_D_{i t}$, para tres submuestras, las integradas por las 8.000, $4.00 \overline{0}$ y 1.000 observaciones representativas de los mayores tamaños, respectivamente.

La tabla 8 recoge los resultados obtenidos para las tres submuestras, que aportan dos interesantes evidencias; la primera de ellas es que la variable $A \tilde{N} O_{t}$ mantiene tanto su signo negativo como su significación, pero en este caso los coeficientes $\beta_{1}$ a ella asociados alcanzan valores de $-0,009,-0,014$ y -0,017 para las submuestras de las $8.000,4.000$ y 1.000 mayores observaciones, en lugar del valor -0,005 que mostramos en la tabla 6 para la muestra completa. Esto significa que la aproximación del tipo efectivo al tipo estatutario se produce con una rapidez mucho mayor en las grandes empresas que en las restantes, y que, además, la convergencia es tanto más rápida cuanto mayor es también el tamaño empresarial.

\begin{tabular}{|c|c|c|c|c|c|c|}
\hline & \multicolumn{2}{|c|}{8000 mayores } & \multicolumn{2}{|c|}{4000 mayores } & \multicolumn{2}{|c|}{1000 mayores } \\
\hline & Coeficientes & $t$ & Coeficientes & $t$ & Coeficientes & $t$ \\
\hline Constante & $-0,198$ & $-1,80^{*}$ & $-0,069$ & $-0,80$ & $-0,272$ & $-1,52$ \\
\hline $\mathrm{AÑ} \mathrm{O}_{\mathrm{t}}$ & $-0,009$ & $-2,72^{* * *}$ & $-0,014$ & $-5,46^{* * *}$ & $-0,017$ & $-3,69^{* * *}$ \\
\hline TAM $_{\text {it }}$ & 0,018 & $2,88^{* * *}$ & 0,023 & $3,18^{* * *}$ & 0,037 & $2,67^{* * *}$ \\
\hline END $_{\text {it }}$ & $-0,076$ & $-1,83^{*}$ & $-0,108$ & $-3,42^{* * *}$ & $-0,063$ & $-1,03$ \\
\hline $\mathrm{INT}_{\mathrm{it}}$ & $-0,097$ & $-1,26$ & $-0,142$ & $-3,02^{* * *}$ & $-0,203$ & $-2,69^{* * *}$ \\
\hline MAT $_{\text {it }}$ & 0,036 & 0,82 & $-0,019$ & $-0,65$ & $-0,068$ & $-1,37$ \\
\hline $\mathrm{ROI}_{\mathrm{it}}$ & 0,395 & $3,76^{* * *}$ & 0,348 & $4,27^{* * *}$ & 0,457 & $3,19^{* * *}$ \\
\hline $\mathrm{TES}_{\mathrm{it}}$ & 0,058 & 0,63 & 0,029 & 0,43 & 0,178 & 0,13 \\
\hline $\mathrm{R}^{2}$ & $11,50 \%$ & & $12,25 \%$ & & $17,12 \%$ & \\
\hline $\mathrm{F}$ & $15,73^{* * *}$ & & $13,12^{* * *}$ & & $10,90^{* * *}$ & \\
\hline $\mathrm{N}$ & 8.000 & & 4.000 & & 1.000 & \\
\hline
\end{tabular}

Tabla 8

Resultados de la regresión del modelo GAP_D $D_{i t}=\alpha+\beta_{1}^{*} \mathrm{ANO}_{t}+$ $\beta_{2}^{*} T A M_{i t}+\beta_{3}^{*} E N D_{i t}+\beta_{4}^{*} I N T_{i t}+\beta_{5}^{*} M A T_{i t}+\beta_{6}^{*} R O I_{i t}+\beta_{7}^{*} T E S_{i t}+\varepsilon_{i t}$

Características de la muestra y definición de variables en la tabla 1. La significación estadística se expresa en asteriscos, a niveles superiores al $90 \%\left({ }^{*}\right), 95 \%\left(^{* *}\right)$ y $99 \%$ $\left(^{* * *}\right)$ de probabilidad. Errores estándar corregidos por dependencia en sección cruzada y serie temporal (Petersen, 2009; Gow et al., 2010). Para simplificar la lectura, no se muestran los coeficientes asociados a sectores económicos.

La segunda evidencia es que la variable $T A M_{i t}$ también mantiene su signo positivo y su significación, pero su coeficiente $\beta_{2}$, que arrojaba un valor de 0,015 en la tabla 6 para la muestra completa, alcanza ahora valores de 0,018, 0,023 y 0,037 para las tres submuestras, lo que implica que, manteniéndose constantes las restantes variables del modelo, el tamaño contribuye a aumentar la diferencia entre el tipo real y el estatutario en un 1,8\%, 2,3\% y 3,7\% con relación a éste último. Por tanto, estos resultados confirman que, si bien en la gran empresa parecen existir mayores oportunidades de planificación fiscal, el proceso de convergencia de los tipos efectivos a los nominales es sensiblemente más acelerado que en las empresas de menor dimensión.

\section{Conclusiones, limitaciones y consideraciones finales}

Sobre una muestra de 31.966 observaciones empresasaños, a lo largo del presente estudio hemos analizado la evolución de la presión fiscal soportada por las empresas españolas en la última década (2008-2017). Nuestros resultados han dejado constancia de una paulatina aproximación de los tipos impositivos reales a los nominales, proceso que es más intenso en el contexto de las empresas de mayor dimensión, que son las que, a tenor de nuestros hallazgos, adoptan estrategias de planificación fiscal más eficientes. Esta realidad pone de manifiesto cómo las medidas adoptadas por la Administración tributaria han dominado con éxito sobre la planificación fiscal, y de aquí que las manifestaciones acerca de la baja tributación de las empresas españolas, señaladamente las de gran tamaño, no parecen estar soportadas por la evidencia que acabamos de documentar.

Nuestro trabajo contribuye al entendimiento de la práctica fiscal de la empresa española y de su evolución temporal. No obstante, nuestros resultados deben interpretarse con cautela; en primer lugar, han de confinarse al ámbito de las empre- 
sas de mayor tamaño, las que formulan cuentas anuales en formato normal, y de aquí que no parezca adecuado extenderlos al contexto de las de una dimensión más reducida, en las que los determinantes de la carga fiscal soportada podrían obedecer a incentivos y circunstancias muy distintos. Nuestro análisis se ha limitado al contexto de las empresas de mayor dimensión como consecuencia, en primer lugar, de que nuestro objetivo ha sido replicar los reproches dirigidos a las grandes empresas -y no a las pequeñas y medianas-sobre su grado de compromiso e implicación fiscal, y en segundo lugar, porque el empleo del tipo impositivo según criterio de caja requiere contar con el estado de flujos de efectivo, que no está disponible en las cuentas anuales que se formulan en modelo abreviado

En segundo lugar, hay que tener muy en cuenta que las medidas de presión fiscal y de convergencia entre tipos reales y nominales que hemos empleado en nuestro análisis empírico son inevitablemente imperfectas, al ser aproximaciones basadas en la información contenida en las cuentas anuales de las empresas y no en microdatos fiscales, que serían los que, en condiciones ideales, permitirían una medición exacta. Así, el ajuste de la doble imposición de los dividendos percibidos que hemos efectuado para calcular los tipos impositivos efectivos, TIE $D_{i t}$ y TIE $C_{i t}$, puede plantear un problema de medición en aquellas observaciones de la muestra en la que la exención no es plena, o en aquellas otras en las que su importe se ha limitado al 50\% de la cuota íntegra, si bien entendemos que ello no afectará de manera sensible a nuestros resultados.

Y en tercer lugar, como es habitual en la literatura empírica sobre planificación fiscal, la evidencia que aportamos debe entenderse referida solamente a empresas con resultados positivos, ya que en nuestra muestra se han excluido aquellas compañías que podrían estar compensando bases imponibles negativas, en las que el tipo efectivo es sensiblemente inferior o incluso cero. Como sostienen Henry \& Sansing (2018), el hecho de trabajar únicamente con compañías en beneficios nos priva de conocer la realidad existente en un importante segmento de la población de empresas, limitando nuestro entendimiento e impidiendo obtener una perspectiva completa de la cuestión.

Por último, nuestro trabajo constituye una primera aportación al entendimiento de la evolución temporal de la presión fiscal de la empresa española, pero dejamos la puerta abierta a futuras extensiones. Así, sería interesante que posteriores contribuciones abordasen esta misma cuestión en el contexto de las pequeñas y medianas empresas, que incorporasen variables representativas de las prácticas de gobierno corporativo en estudios confinados a las compañías cotizadas-que es otro subconjunto de empresas de gran interés-o que mostrasen posibles diferencias regionales en prácticas de planificación fiscal, para ilustrar el posible impacto de regímenes fiscales forales o insulares y el posible efecto de diferentes grados de enforcement de las administraciones territoriales.

\section{Agradecimiento}

Esta investigación no recibió ninguna subención específica de organismos de financiación de los sectores público, clomercial o sin ánimo de lucro.

\section{Conflicto de intereses}

Los autores declaran no tener nignún conflicto de intereses.

\section{Bibliografía}

AEDAF (2017). Los tipos efectivos del Impuesto sobre Sociedades en España. Asociación Española de Asesores Fiscales, Madrid, marzo.

Álvarez García, S., Fernández Rodríguez, E., \& Martínez Arias, A. (2004). El tipo impositivo contable y fiscal de las sociedades españolas a lo largo de la década de los noventa. Partida Doble, 153, 60-67.

Armstrong, C., Blouin, J., \& Larcker, D.F. (2012). Incentives for tax planning. Journal of Accounting and Economics, 53, 391-411. https://doi.org/10.1016/j.jacceco.2011.04.001

Armstrong, C., Blouin, J., Jagolinzer, J., \& Larcker, D.F. (2015). Corporate governance, incentives, and tax avoidance. Journal of Accounting and Economics, 60, 1-17. https: //doi.org/10.1016/j.jacceco.2015.02.003

Badertscher, B., Katz, S., \& Rego, S. (2013). The separation of ownership and control and corporate tax avoidance. Journal of Accounting and Economics, 56, 228-250. https: //doi.org/10.1016/j.jacceco.2013.08.005

Blouin, J. (2014). Defining and measuring tax planning aggressiveness. National Tax Journal, 67, 875-900. DOI, dx.doi.org/10.17310/ntj.2014.4.06

Bona-Sánchez, C., Pérez-Alemán, J., \& Santana Martín, D.J. (2019). Political ties and corporate tax burden in Spain. Revista Española de Financiación y Contabilidad, 49, 74-93. https://doi.org/10.1080/02102412.2019.1573049

Calvé Pérez, J. I., Labatut Serer, G., \& Molina Llopis, R. (2005). Variables económico-financieras que inciden sobre la presión fiscal soportada por las empresas de ńreducida dimensiónż, Efectos de la reforma fiscal en las empresas de la Comunidad Valenciana. Revista Española de Financiación y Contabilidad, 34, 875-897. https://doi.org/10.1080/ 02102412.2005.10779565

Chen, S., Chen, X., Cheng, Q., \& Shevlin, T. (2010). Are family firms more tax aggressive than non-family firms?. Journal of Financial Economics, 95, 41-61. https://doi.org/10. 1016/j.jfineco.2009.02.003

CEOE (2017). Informe sobre impuestos y empresas. Confederación Española de Organizaciones Empresariales, Madrid, febrero.

Conde-Ruiz, J. I. (2015). Reflexiones sobre la fiscalidad de las grandes empresas multinacionales en España. FEDEA, documento de trabajo No. 2015-06. https://ideas.repec.org/s/ fda/fdapop.html

Davis, A., Guenther, D., Krull, L., \& Williams, B. (2016). Do socially responsible firms pay more taxes? Accounting Review, 91, 47-68. https://doi.org/10.2308/accr-51224

De Angelo, H., \& Masulis, R.W. (1980). Optimal capital structure under corporate and personal taxation. Journal of Financial Economics, 8, 3- 29. https://doi.org/10.1016/ 0304-405X(80)90019-7

De Simone, L., Nickerson, J., Seidman, J. K., \& Stomberg, B. (2019). How reliably do empirical tests identify tax avoidance? Contemporary Accounting Research, en prensa. https: //doi.org/10.1111/1911-3846.12573

Desai, M., \& Dharmapala, D. (2006). Corporate tax avoidance and high-powered incentives. Journal of Financial Economics, 79, 145-179. https://doi.org/10.1016/j.jfineco. 2005.02.002

Desai, M.A., Dyck, A., \& Zingales, L. (2007). Theft and taxes. Journal of Financial Economics, 84, 591-623. https: //doi.org/10.1016/j.jfineco.2006.05.005

Drake, K., Hamilton, R., \& Lusch, S. J. (2018). The sources of declining effective tax rates for multinational and domestic firms, Insight from effective tax rate reconciliations. 
Documento de trabajo, University of Arizona.

Dyreng, S.D., Hanlon, M., \& Maydew, E.L. (2008). Longrun corporate tax avoidance. Accounting Review, 83, 61-82. https://doi.org/10.2308/accr.2008.83.1.61

Dyreng, S., Hoopes, J., \& Wilde, J. (2016). Public pressure and corporate tax behavior. Journal of Accounting Research, 54, 147-186. https://doi.org/10.1111/1475-679X.12101

Dyreng, S.D., Hanlon, M., Maydew, E.L., \& Thornock, J.R. (2017). Changes in corporate effective tax rates over the past 25 years. Journal of Financial Economics, 124, 441-463. https: //doi.org/10.1016/j.jfineco.2017.04.001

Edwards, A., Kubata, A., \& Shevlin, T. (2019). The decreasing trend in cash effective tax rates, The role of growth in pre-tax income. Documento de trabajo, University of California at Irvine, abril.

Feito, J.L. (2018). £Deben bajar o subir los impuestos en España?. Documento de trabajo, Instituto de Estudios Económicos, Madrid, diciembre.

Fernández Rodríguez, E. (2002). El tipo positivo efectivo (TIE) como indicador de la presión fiscal empresarial. Aplicación práctica sobre las empresas cotizadas. Revista Técnica Tributaria, 59, 27-70.

Fernández Rodríguez, E. (2004a). Los factores condicionantes de la presión fiscal empresarial española a partir de la información contable. Especial mención a las decisiones financieras. Revista Española de Financiación y Contabilidad, 120, 125-159. https://doi.org/10.1080/02102412. 2004.10779516

Fernández Rodríguez, E. (2004b). La imposición efectiva de las sociedades españolas desde la óptica contable y fiscal. Instituto de Estudios Fiscales, Madrid. ISBN 84-8008-168-6.

Fernández Rodríguez, E., \& Martínez Arias, A. (2008a). La presión fiscal del Impuesto sobre Sociedades por sectores de actividad. Crónica Tributaria, 127, 27-58.

Fernández Rodríguez, E., \& Martínez Arias, A. (2008b). La presión fiscal del Impuesto sobre Sociedades por comunidades autónomas. Técnica Tributaria, 82, 23-47.

Fonseca Díaz, A. R., Fernández Rodríguez, E., \& Martínez Arias, A. (2011). Factores condicionantes de la presión fiscal de las entidades de crédito españolas. $£$ Existen diferencias entre bancos y cajas de ahorros? Revista Española de Financiación y Contabilidad, 40, 491-516. https://doi.org/10.1080/ 02102412.2011.10779710

Fernández Rodríguez, E., García Fernández, R., \& Martínez Arias, A. (2019). Influence of ownership structure on the determinants of effective tax rates of Spanish companies. Sustainability, 11, 1441. http,//dx.doi.org/10.3390/su11051441

Frank, M.M., Lynch, L.J., \& Rego, S.O. (2009). Tax reporting aggressiveness and its relation to aggressive financial reporting. Accounting Review, 84, 467-496. https://doi.org/10. 2308/accr.2009.84.2.467

Gallemore, J., \& Labro, E. (2015). The importance of the internal information environment for tax avoidance. Journal of Accounting and Economics, 60, 149-167. https://doi.org/ 10.1016/j.jacceco.2014.09.005

Gallemore, J., Maydew, E. L., \& Thornock, J. R. (2014). The reputational costs of tax avoidance. Contemporary Accounting Research, 31, 1103-1133. https://doi.org/10.1111/ 1911-3846.12055

Gow, I.D., Ormazábal, G., \& Taylor, D.J. (2010). Correcting for cross-sectional and time-series dependence in accounting research. Accounting Review, 85, 483-512. http,//dx.doi.org/10.2308/accr.2010.85.2.483

Graham, J.R., Raedy, J.S., \& Shackelford, D.A. (2012). Research in accounting for income taxes. Journal of Accounting and Economics, 53, 412-434. https://doi.org/10.1016/

\section{j.jacceco.2011.11.006}

Guenther, D. A., Wilson, R. J., \& Wu, K. (2019). Tax uncertainty and incremental tax avoidance. Accounting Review, 94, 229-247. https://doi.org/10.2308/accr-52194

Gupta, S., \& Newberry, K. (1997). Determinants of the variability in corporate effective tax rates, Evidence from longitudinal data. Journal of Accounting and Public Policy, 16, 1-34. https://doi.org/10.1016/S0278-4254(96)00055-5

Hanlon, M. (2003). What can we infer about a firm's taxable income from its financial statements? National Tax Journal, 56, 831-863. http,//dx.doi.org/10.17310/ntj.2003.4.07

Hanlon, M., \& Heitzman, S. (2010). A review of tax research. Journal of Accounting and Economics, 50, 127-178. https://doi.org/10.1016/j.jacceco.2010.09.002

Hanlon, M., Maydew, E. L., \& Saavedra, D. (2013). Understanding why firms hold so much cash, A tax risk explanation. En 2013 American Taxation Association Midyear Meeting, Uncertain Tax Positions.

Henry, E., \& Sansing, R. (2018). Corporate tax avoidance, data truncation and loss firms. Review of Accounting Studies, 23, 1042-1070. https://doi.org/10.1007/ s11142-018-9448-0

Higgins, D., Omer, T. C., \& Phillips, J. D. (2015). The influence of a firm's business strategy on its tax aggressiveness.Contemporary Accounting Research, 32, 674-702. https: //doi.org/10.1111/1911-3846.12087

Kanagaretnam, K., Lee, J. Lim, C.Y., \& Lobo, G.J. (2016). Relation between auditor quality and corporate tax aggressiveness, Implications of cross-country institutional differences, Auditing, A Journal of Practice and Theory, 35, 105-135. https://doi.org/10.2308/ajpt-51417

Lennox, C., Lisowsky, P., \& Pittman, J. (2012). Tax aggressiveness and accounting fraud. Journal of Accounting Research, 51, 739-778. https://doi.org/10.1111/joar.12002

Manzon, G.B., \& Plesko, G.A. (2002). The relation between financial and tax reporting measures of income. Tax Law Review, 55, 175-214.

Modigliani, F., \& Miller, M. (1963). Corporate income taxes and the cost of capital, A correction. American Economic Review, 53, 433-443. https://www-jstor-org.ezproxy.unex.es/ stable/1809167

Molina Llopis, R. (2005). Presión fiscal en las pymes. Estudio de su incidencia en la Comunidad Valenciana. Asociación Española de Contabilidad y Administración de Empresas, Madrid. ISSN, 84-89959-91-9

Monterrey Mayoral, J., \& Sánchez Segura, A. (2010). Diferencias en agresividad fiscal entre empresas familiares y no familiares. Revista Española de Financiación y Contabilidad, 39, 65-107. https://doi.org/10.1080/02102412.2010. 10779679

Monterrey Mayoral, J., \& Sánchez Segura, A. (2015). Planificación fiscal y Gobierno Corporativo en las empresas cotizadas españolas. Hacienda Pública Española, 214, 55-89. http,//doi.org/10.7866/HPE-RPE.15.3.3

Moreno Rojas, J., González Rodríguez, M.R., \& Martín Samper, R.C. (2017). Determinants of the effective tax rate in the tourism sector, a dynamic panel data model. Tourism \& Management Studies, 13, 3138. http,//doi.org/10.18089/tms.2017.13304

OCDE (2003). Using micro-data to asses average tax rates. OECD Tax Policy Studies, No. 8, París. https://doi.org/10. $1787 / 19900538$

Petersen, M. (2009). Estimating standard errors in finance panel data sets. Comparing approaches. Review of Financial Studies, 22, 435-480. https://doi.org/10.1093/rfs/hhn053

Richardson, G., Taylor, G., \& Lanis, R. (2013). The impact 
of board of director oversight characteristics on corporate tax aggressiveness, An empirical analysis. Journal of Accounting and Public Policy, 32, 68-88. https://doi.org/10.1016/j. jaccpubpol.2013.02.004

Scholes, M., Wolfson, M.A., Erickson, M., Hanlon, M.L., Maydew, E.L., \& Shevlin, T. (2015). Taxes and Business Strategy, A Planning Approach. Prentice Hall, $5 \nmid$ edición, Upper Saddle River, NJ. ISSN, 978-0130253989.

Shackelford, D.A., \& Shevlin, T. (2001). Empirical tax research in Accounting. Journal of Accounting and Economics, 31, 321-387. https://doi.org/10.1016/S0165-4101(01) $00022-2$ 